TID - 5360 (Suppl. 5)

HEALTH AND SAFETY

(TID $-4500,47$ th. Ed.)

\title{
A SUMMARY OF
}

\section{INDUSTRIAL ACCIDENTS IN USAEC FACILITIES}

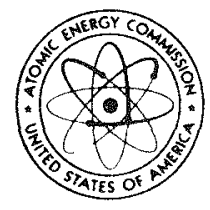

UNITED STATES ATOMIC ENERGY COMMISSION

Safety and Fire Protection Branch Office of Industrial Relations 


\section{DISCLAIMER}

This report was prepared as an account of work sponsored by an agency of the United States Government. Neither the United States Government nor any agency Thereof, nor any of their employees, makes any warranty, express or implied, or assumes any legal liability or responsibility for the accuracy, completeness, or usefulness of any information, apparatus, product, or process disclosed, or represents that its use would not infringe privately owned rights. Reference herein to any specific commercial product, process, or service by trade name, trademark, manufacturer, or otherwise does not necessarily constitute or imply its endorsement, recommendation, or favoring by the United States Government or any agency thereof. The views and opinions of authors expressed herein do not necessarily state or reflect those of the United States Government or any agency thereof. 


\section{DISCLAIMER}

Portions of this document may be illegible in electronic image products. Images are produced from the best available original document. 
$\mid$

-

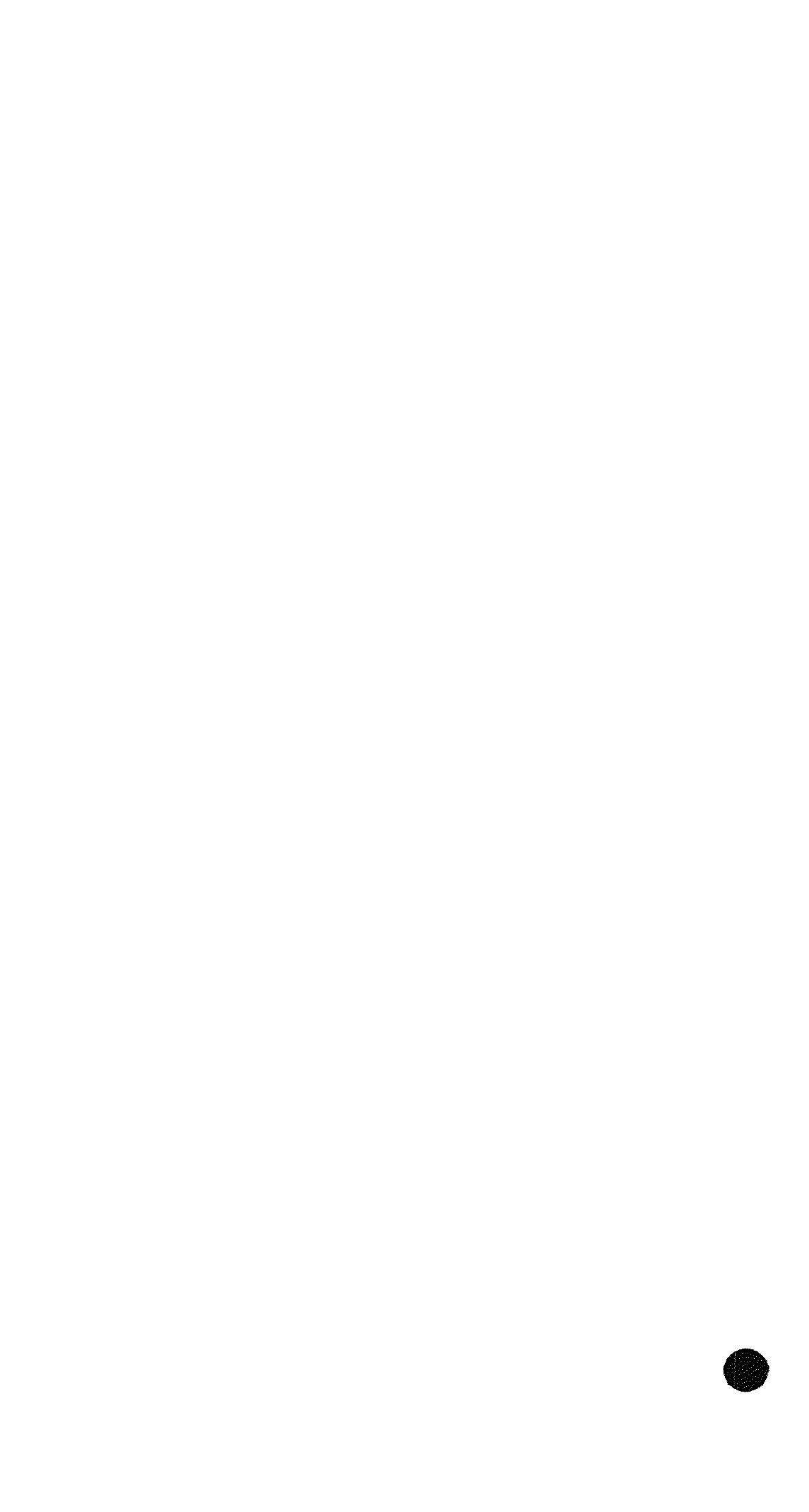

.
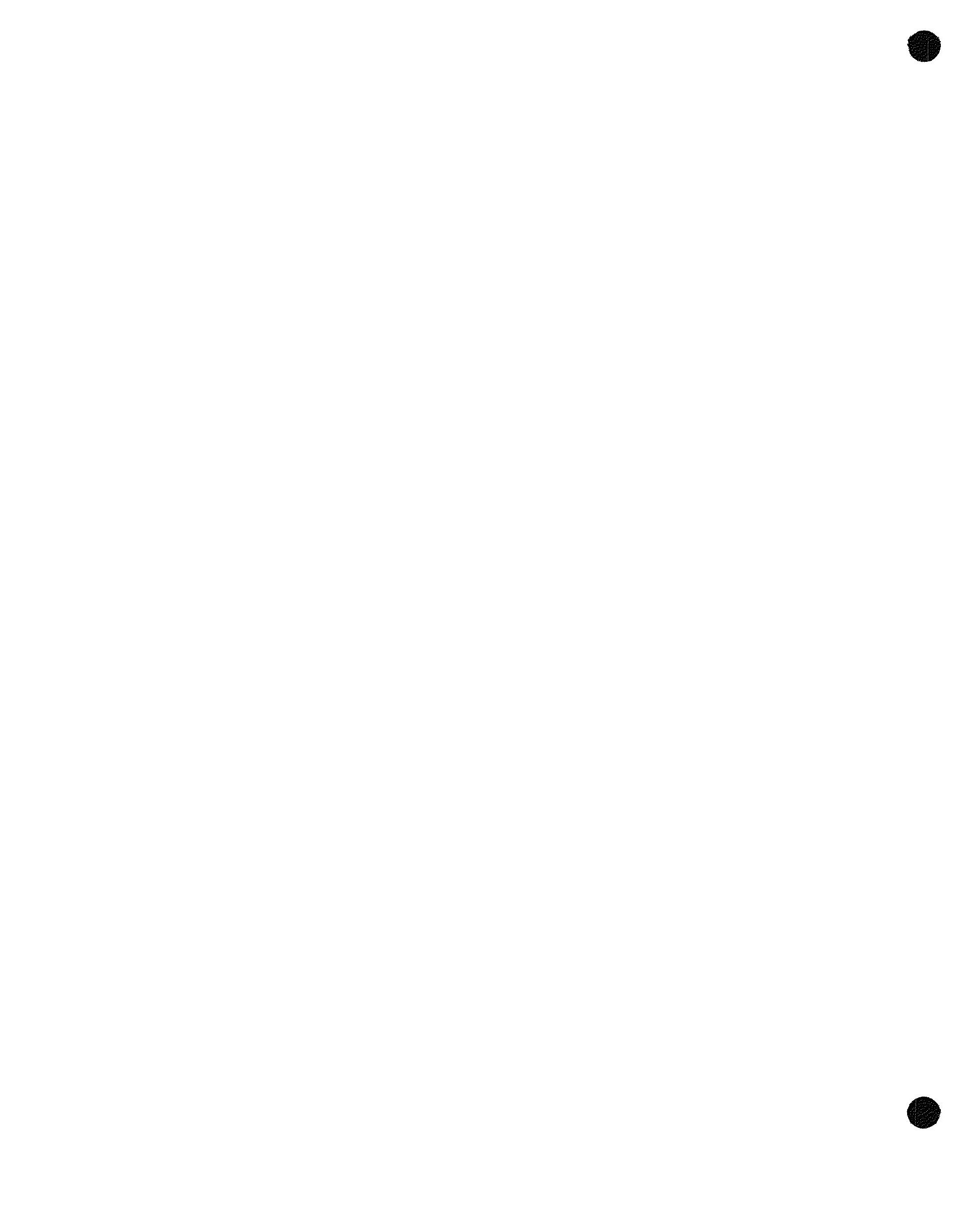


\section{PREFACE}

"A Summary of Industrial Accidents in USAEC Facilities," TID-5360 (Suppl. 5), presents information on accidents and incidents occurring during 1963-64 in plants owned and operated by the Atomic Energy Commission. The original TID- 5360 covered the years $1945-55$, the first supplement 1956 , the second supplement 1957-58; the third supplement $1959-60$, and the fourth supplement $1961-62$.

This Summary does not contain accident experience in private or licensed operations.

Short narrative descriptions of accidents and incidents involving radioactive materials, together with a table of radiation exposures for AEC contractor personnel, are included because of their special interest to the atomic energy industry. Also included are descriptions of industrial fatal accidents.

Reporting requirements are contained in AEC' Chapter 0502, "Reporting and Investigating Accidents and Radiation Exposures." This chapter categorizes types of accidents and radiation exposures and designates them as "Type A" and "Type B," which are defined in this publication at the conclusion of the listing entitled "Industrial Accidents and Radiation Exposure Incidents." 


\section{CONTENTS}

Page

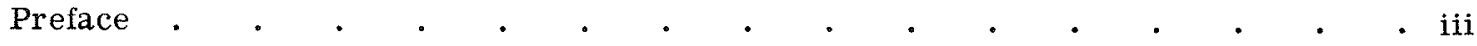

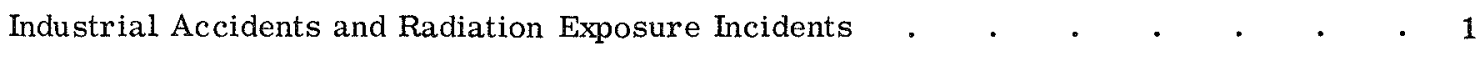

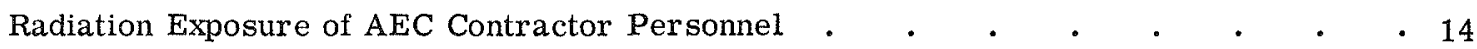

Accidents and Incidents in AEC Facilities Involving Radioactive Material

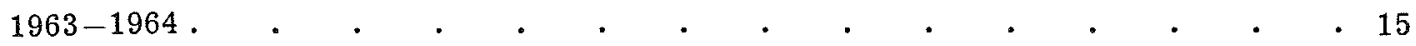

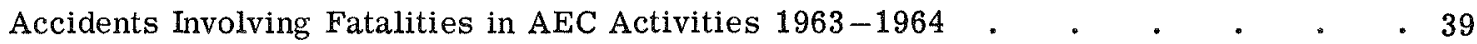




\section{Industrial Accidents and Radiation Exposure Incidents}

USAEC Facilities, 1963-1964

\begin{tabular}{|c|c|c|c|c|c|}
\hline $\begin{array}{l}\text { No.* \& } \\
\text { Type } \dagger\end{array}$ & Date & Field Office \& Contractor & $\begin{array}{r}\text { Injuries } \$ \text { \& } \\
\text { Exposures }\end{array}$ & $\begin{array}{l}\text { AEC Property } \\
\text { Damage }\end{array}$ & Remarks \\
\hline & 1963 & & & & \\
\hline $63-1 \mathrm{~A}$ & $1-1$ & ID-Phillips Petroleum Co. & 0 & 0 & $\begin{array}{l}\text { Truck transporting fuel } \\
\text { elements involved in ac- } \\
\text { cident with private car. } \\
\text { Shipment undamaged. }\end{array}$ \\
\hline $63-2 \mathrm{~A}$ & $1-5$ & $\mathrm{CH}$-Atomics International & 1 killed & 0 & $\begin{array}{l}\text { Shift supervisor died of in- } \\
\text { ternal injuries and basal } \\
\text { skull fracture resulting } \\
\text { from } 54^{\prime} \text { fall when he } \\
\text { stepped backward into } \\
\text { open shield plug hole } \\
\text { while giving signals to } \\
\text { crane operator. (See } \\
\text { page 39). }\end{array}$ \\
\hline $63-3 \mathrm{~A}$ & $1-10$ & $\begin{array}{l}\text { OR-Mallinckrodt Chemical } \\
\text { Works }\end{array}$ & 0 & 0 & $\begin{array}{l}\text { Truck transporting radio- } \\
\text { active material involved } \\
\text { in accident with private } \\
\text { car. Shipment undamaged. }\end{array}$ \\
\hline $63-4 \mathrm{~A}$ & $1-10$ & $\begin{array}{l}\text { OR-National Lead Co. of } \\
\text { Ohio }\end{array}$ & 0 & 0 & $\begin{array}{l}\text { Truck transporting ura- } \\
\text { nium fuel slugs involved } \\
\text { in accident with private } \\
\text { car. Shipment undamaged. }\end{array}$ \\
\hline $63-5 \mathrm{~A}$ & $1-14$ & $\begin{array}{l}\text { BH-NY-Nuclear Materials } \\
\text { \& Equipment Corp. }\end{array}$ & 0 & 27,560 & $\begin{array}{l}\text { One of eleven packages of } \\
\text { contaminated precious } \\
\text { metals found leaking upon } \\
\text { arrival at destination. } \\
\text { Decontamination of sev- } \\
\text { eral vehicles and truck } \\
\text { depots necessitated. (See } \\
\text { page 15). }\end{array}$ \\
\hline $63-6 \mathrm{~B}$ & $1-24$ & OR-Government & 0 & 6,787 & $\begin{array}{l}\text { Government-owned dwelling } \\
\text { totally destroyed by fire } \\
\text { believed started in defec- } \\
\text { tive heating system. }\end{array}$ \\
\hline $63-7 \mathrm{~B}$ & $1-26$ & PNR-Duquesne Light Co. & 0 & 33,455 & $\begin{array}{l}\text { Fire (definite cause unde- } \\
\text { termined) occurred in } \\
\text { contractor-owned office } \\
\text { building. Government- } \\
\text { owned office machines, } \\
\text { furniture and other equip- } \\
\text { ment damaged. }\end{array}$ \\
\hline $63-11 \mathrm{~A}$ & $2-16$ & NV-Camay Drilling Co. & 1 killed & 0 & $\begin{array}{l}\text { During excavation work, a } \\
\text { construction worker was } \\
\text { crushed by a tractor when } \\
\text { the entire length of the } \\
\text { tractor tread ran over } \\
\text { his body, causing multi- } \\
\text { ple internal injuries. } \\
\text { (See page } 40 \text { ). }\end{array}$ \\
\hline $63-12 \mathrm{~A}$ & $2-12$ & RL-General Electric Co. & 0 & 8,100 & $\begin{array}{l}\text { Promethium } 147 \text { contami- } \\
\text { nation found in laboratory } \\
\text { rooms; also tracked to } \\
\text { other parts of building. } \\
\text { (See page 16). }\end{array}$ \\
\hline $63-13 \mathrm{~B}$ & $2-10$ & A L-Sandia Corp. & 0 & 10,200 & $\begin{array}{l}\text { During construction of lab- } \\
\text { oratory, power outage } \\
\text { resulted in deflation of } \\
\text { "air structure,", which } \\
\text { was completely destroyed } \\
\text { by high winds. }\end{array}$ \\
\hline
\end{tabular}


INDUSTRIAL ACCIDENTS AND RADIATION EXPOSURE INCIDENTS (Cont'd)

\begin{tabular}{|c|c|c|c|c|c|}
\hline $\begin{array}{l}\text { No.* \& } \\
\text { Type } \dagger\end{array}$ & Date & Field Office \& Contractor & $\begin{array}{c}\text { Injuries李 \& } \\
\text { Exposures }\end{array}$ & $\begin{array}{c}\text { AEC Property } \\
\text { Damage }\end{array}$ & Remarks \\
\hline $63-14 \mathrm{~B}$ & $2-3$ & RL-Government & 0 & $\$ 15,350$ & $\begin{array}{l}\text { Two small earth-f } 11 \text { dams } \\
\text { washed out by melting } \\
\text { snow. }\end{array}$ \\
\hline $63-15 \mathrm{~B}$ & $2-26$ & $\begin{array}{l}\text { SR-E. I. du Pont } \\
\text { de Nemours \& Co. }\end{array}$ & 0 & 24,000 & $\begin{array}{l}\text { Fuel element dropped into } \\
\text { reactor, damaging ele- } \\
\text { ment. }\end{array}$ \\
\hline $63-16 \mathrm{~B}$ & $3-14$ & $\begin{array}{l}\text { SR-E. I. du Pont } \\
\text { de Nemours \& Co. }\end{array}$ & 0 & & $\begin{array}{l}\text { Tritium release to atmo- } \\
\text { sphere through stack. } \S \\
\text { (See page } 16 \text { ). }\end{array}$ \\
\hline $63-17 \mathrm{~A}$ & $3-26$ & $\begin{array}{l}\text { SAN-Lawrence Radiation } \\
\text { Laboratory }\end{array}$ & 0 & 94,881 & $\begin{array}{l}\text { Criticality occurred during } \\
\text { subcritical experiment } \\
\text { involving fissionable ma- } \\
\text { terials and small fire de- } \\
\text { veloped within enclosed } \\
\text { concrete vault. (See } \\
\text { page 17). }\end{array}$ \\
\hline $63-18 \mathrm{~B}$ & $3-16$ & AL-Dow Chemical Co & 0 & 8,200 & $\begin{array}{l}\text { High-velocity winds caused } \\
\text { circuit breaker failure in } \\
\text { substation, resulting in } \\
\text { fire readily controlled by } \\
\text { fire extinguisher. }\end{array}$ \\
\hline $63-19 \mathrm{~B}$ & $1-12$ & ID-C. F. Braun \& Co. & 0 & 5,800 & $\begin{array}{l}\text { During subzero weather, } \\
\text { water in firewater header } \\
\text { froze, damaging header. }\end{array}$ \\
\hline $63-20 \mathrm{~B}$ & $4-17$ & RL-General Electric Co. & 1 exposed & 0 & $\begin{array}{l}\text { Maintenance employee re- } \\
\text { celved whole-body expo- } \\
\text { sure of } 5 \text { rem while work- } \\
\text { ing on fuel tube of reactor } \\
\text { (See page 17). }\end{array}$ \\
\hline $63-21 \mathrm{~A}$ & $4-22$ & $\begin{array}{l}\text { OR-Union Carbide Corp., } \\
\text { Nuclear Div. }\end{array}$ & 0 & 25 & $\begin{array}{l}\text { Tractor-trailer carrying } \\
\text { interplant shipment of } \\
\text { radioactive materials } \\
\text { tipped over when forced } \\
\text { off road by oncoming traf- } \\
\text { fic. Shipment undamaged. }\end{array}$ \\
\hline $63-22 \mathrm{~B}$ & $4-20$ & NV-Sandia Corp. & 0 & 14,848 & $\begin{array}{l}\text { Fire damaged aerodynamic } \\
\text { balloon when gasoline } \\
\text { overflowed from genera- } \\
\text { tor engine and was ignited } \\
\text { by engine backfire. Later, } \\
\text { on same day, high-veloc- } \\
\text { 1ty winds completely } \\
\text { destroyed balloon. }\end{array}$ \\
\hline $63-23 \mathrm{~B}$ & $4-25$ & NV-Government & 0 & 12,000 & $\begin{array}{l}\text { High-velocity winds dam- } \\
\text { aged three balloons. }\end{array}$ \\
\hline $63-24 \mathrm{~A}$ & $4-30$ & $\begin{array}{l}\text { OR-Union Carbide Corp., } \\
\text { Nuclear Div. }\end{array}$ & $1 \mathrm{k}$ lled & 0 & $\begin{array}{l}\text { While testing an ion source, } \\
\text { a physicist was elec- } \\
\text { trocuted. (See page } 40 \text { ). }\end{array}$ \\
\hline $63-25 \mathrm{~B}$ & $5-16$ & RL-Ka1ser Engineers & 5 exposed & 0 & $\begin{array}{l}\text { During process of remov- } \\
\text { ing control cables from } \\
\text { radiographic camera, } 27- \\
\text { curie iridium } 192 \text { sealed } \\
\text { source fell out of cable } \\
\text { and remained unnoticed on } \\
\text { cell floor for approxi- } \\
\text { mately five hours. Dur- } \\
\text { ing this period, approxi- } \\
\text { mately } 76 \text { workers were } \\
\text { 1ntermittently in area } \\
\text { where exposed source } \\
\text { was located. Five individ- } \\
\text { uals exceeded } 3.0 \text { rem } \\
\text { per quarter, highest being } \\
15 \text { rem. (See page 19) }\end{array}$ \\
\hline
\end{tabular}


INDUSTRIAL ACCIDENTS AND RADIATION EXPOSURE INCIDENTS (Cont'd.)

\begin{tabular}{|c|c|c|c|c|c|}
\hline $\begin{array}{l}\text { No.* \& } \\
\text { Type } \dagger\end{array}$ & Date & Field Office \& Contractor & $\begin{array}{c}\text { Injuriest \& } \\
\text { Exposures }\end{array}$ & $\begin{array}{l}\text { AEC Property } \\
\text { Damage }\end{array}$ & Remarks \\
\hline $63-26 \mathrm{~A}$ & $6-6$ & $\begin{array}{l}\text { NV-Reynolds Electrical } \\
\text { \& Engineering Co., Inc. }\end{array}$ & 13 exposed & 0 & $\begin{array}{l}\text { Fifteen employees exposed } \\
\text { during re-entry and re- } \\
\text { covery operation in tun- } \\
\text { nel, thirteen receiving in } \\
\text { excess of } 30 \text { rem per } \\
\text { year to thyroid. (See } \\
\text { page 19). }\end{array}$ \\
\hline $63-27 \mathrm{~B}$ & $\begin{array}{r}4-15 / \\
5-8\end{array}$ & CH-Atomics International & 1 exposed & 0 & $\begin{array}{l}\text { Employee received partial- } \\
\text { body exposure of } 11 \text { rem } \\
\text { while working on } \mathrm{X}-\mathrm{ray} \\
\text { diffraction machine. (See } \\
\text { page 17). }\end{array}$ \\
\hline $63-28 \mathrm{~B}$ & $6-19$ & $\begin{array}{l}\text { NV-Reynolds Electrical } \\
\text { \& Engineering Co., Inc. }\end{array}$ & 0 & 17,000 & $\begin{array}{l}\text { Drill rig toppled over while } \\
\text { leveling adjustments } \\
\text { were in process. }\end{array}$ \\
\hline $63-29 \mathrm{~A}$ & $6-25$ & SNR-Marley Corp. & 1 killed & 0 & $\begin{array}{l}\text { During construction of fan } \\
\text { cylinder, carpenter fell } \\
65^{\prime} \text {. Cause of death was } \\
\text { compound fractured skull, } \\
\text { broken neck and multiple } \\
\text { injuries with massive } \\
\text { hemorrhage. (See page } \\
40) \text {. }\end{array}$ \\
\hline $63-30 \mathrm{~A}$ & $6-25$ & $\begin{array}{l}\text { NY-Pratt \& Whitney Air- } \\
\text { craft Div., CANEL } \\
\text { Operations }\end{array}$ & 1 killed & 0 & $\begin{array}{l}\text { While operator's helper } \\
\text { was kneeling on top of } \\
\text { box, being moved by fork- } \\
\text { lift truck, to counter- } \\
\text { balance overhanging } \\
\text { weight, box tipped for- } \\
\text { ward and he fell to ground. } \\
\text { He suffered fatal pulmo- } \\
\text { nary embolus seventeen } \\
\text { days later as result of } \\
\text { injuries received in acci- } \\
\text { dent. (See page } 41 \text { ). }\end{array}$ \\
\hline $63-31 \mathrm{~B}$ & $4-23$ & AL-Dow Chemical Co. & 0 & 5,662 & $\begin{array}{l}\text { Spill of contaminated nitric } \\
\text { acid solution. (See page } \\
\text { 18). }\end{array}$ \\
\hline $63-32 \mathrm{~B}$ & $6-20$ & AL-Dow Chemical Co. & 0 & 8,364 & $\begin{array}{l}\text { Leak in line carrying high- } \\
\text { level plutonium solution } \\
\text { caused contamination of } \\
\text { building and equipment. } \\
\text { (See page 20). }\end{array}$ \\
\hline $63-34 \mathrm{~B}$ & $8-9$ & $\begin{array}{l}\text { PNR-Westinghouse Elec- } \\
\text { tric Corp. }\end{array}$ & 0 & 9,400 & $\begin{array}{l}\text { Severe winds during elec- } \\
\text { trical storm damaged } \\
\text { roofs, stacks, ventilation } \\
\text { ducts, trees, shrubs and } \\
\text { fences. }\end{array}$ \\
\hline $63-35 \mathrm{~B}$ & $\begin{array}{r}6-28 / \\
7-8\end{array}$ & $\begin{array}{l}\text { SAN-Lawrence Radiation } \\
\text { Laboratory }\end{array}$ & 2 exposed & 0 & $\begin{array}{l}\text { Two employees received } \\
\text { quarterly exposures to } \\
\text { their right hands in ex- } \\
\text { cess of } 25 \text { rem, (calendar } \\
\text { year exposures of } 72.9 \\
\text { and } 64.2 \text { rem) while en- } \\
\text { gaged in transferring ra- } \\
\text { dioactive materials into } \\
\text { high vacuum chamber } \\
\text { using short tongs. (See } \\
\text { page } 20) \text {. }\end{array}$ \\
\hline $63-36 \mathrm{~A}$ & $7-23$ & OR-H. K. Ferguson Co. & 1 killed & 0 & $\begin{array}{l}\text { Apprentice pipefitter was } \\
\text { asphyxiated while check- } \\
\text { ing for inert gas leak in } \\
\text { valve pit. (See page } 42 \text { ). }\end{array}$ \\
\hline $63-37 \mathrm{~A}$ & $7-24$ & $\begin{array}{l}\text { AL-Mason \& Hanger-Silas } \\
\text { Mason Co., Inc., San } \\
\text { Antonio, Tex. }\end{array}$ & 0 & 0 & $\begin{array}{l}\text { Train derailed. Coach } \\
\text { carrying radioactive ma- } \\
\text { terials remained upright. } \\
\text { Shipment undamaged. }\end{array}$ \\
\hline
\end{tabular}


INDUSTRIAL ACCIDENTS AND RADIATION EXPOSURE INCIDENTS (Cont'd.)

\begin{tabular}{|c|c|c|c|c|c|}
\hline $\begin{array}{r}\text { No.* \& } \\
\text { Type } †\end{array}$ & Date & Field Office \& Contractor & $\begin{array}{c}\text { Injuriest \& } \\
\text { Exposures }\end{array}$ & $\begin{array}{l}\text { AEC Property } \\
\text { Damage }\end{array}$ & Remarks \\
\hline $63-38 \mathrm{~B}$ & $\begin{array}{l}\text { Apr.-- } \\
\text { June }\end{array}$ & OR-General Electric Co. & 2 exposed & 0 & $\begin{array}{l}\text { Two employees received } \\
\text { quarterly external radia- } \\
\text { tion exposures, one } 3.9 \\
\text { rem whole body, } 32 \text { rem } \\
\text { left hand, } 14 \text { rem right } \\
\text { hand; the other, } 2.1 \text { rem } \\
\text { whole body, } 27 \text { rem left } \\
\text { hand, } 25 \text { rem left hand. } \\
\text { Exposures occurred while } \\
\text { making physical measure- } \\
\text { ments of irradiated beryl- } \\
\text { lium oxide samples. (See } \\
\text { page 18). }\end{array}$ \\
\hline $63-39$ A & $8-3$ & $\begin{array}{l}\text { PNR-Westinghouse Elec- } \\
\text { tric Corp. }\end{array}$ & 0 & 0 & $\begin{array}{l}\text { Shipping cask dropped dur- } \\
\text { ing transfer. Contamina- } \\
\text { tion removed from } 1 / 2- \\
\text { square foot spot by } \\
\text { removing } 1 / 8 \text { th-inch of } \\
\text { wooden surface. (See } \\
\text { page } 22 \text { ). }\end{array}$ \\
\hline $63-40 \mathrm{~B}$ & $8-6$ & $\begin{array}{l}\text { OR-Union Carbide Corp. } \\
\text { Nuclear Division }\end{array}$ & 0 & 43,400 & $\begin{array}{l}\text { Fire (definite cause unde- } \\
\text { termined) originated in } \\
\text { building exhaust system } \\
\text { and was confined to lab- } \\
\text { oratory hoods and exhaust } \\
\text { system; however, smoke } \\
\text { damaged building. }\end{array}$ \\
\hline $63-41 \mathrm{~A}$ & $8-1$ & $\begin{array}{l}\text { OR-Union Carbide Corp. } \\
\text { Paducah, Ky. }\end{array}$ & 1 killed & 0 & $\begin{array}{l}\text { While ironworker was } \\
\text { climbing down rolling- } \\
\text { type scaffold, scaffold } \\
\text { began to swing and fold, } \\
\text { finally toppling to floor. } \\
\text { He held on until scaffold } \\
\text { was about halfway down, } \\
\text { then fell remaining dis- } \\
\text { tance (approximately } 6^{\prime} \text { ) } \\
\text { to concrete floor, dying } \\
\text { five days later as result } \\
\text { of skull fracture. (See } \\
\text { page } 42 \text { ). }\end{array}$ \\
\hline $63-43 \mathrm{~B}$ & $7-23$ & $\begin{array}{l}\text { SR-E. I. du Pont } \\
\text { de Nemours \& Co. }\end{array}$ & 0 & & $\begin{array}{l}\text { Tritium release to atmo- } \\
\text { sphere through stack. } \\
\text { (See page } 21 \text { ). }\end{array}$ \\
\hline $63-44 \mathrm{~B}$ & $7-25$ & NY-Babcock \& Wilcox Co. & 1 exposure & 0 & $\begin{array}{l}\text { While engaged in routine } \\
\text { radiography, employee } \\
\text { received estimated expo- } \\
\text { sure of } 6 \text { rem. (See } \\
\text { page } 21 \text { ). }\end{array}$ \\
\hline $63-45 \mathrm{~A}$ & $7-10$ & $\begin{array}{l}\text { NV-Reynolds Electrical } \\
\text { \& Engineering Co., Inc. }\end{array}$ & 1 killed & 0 & $\begin{array}{l}\text { A custodial employee suf- } \\
\text { fered fatal pulmonary } \\
\text { embolism as result of in- } \\
\text { juries received in auto- } \\
\text { mobile accident three } \\
\text { months earlier. (See } \\
\text { page } 41 \text { ). }\end{array}$ \\
\hline $63-46 \mathrm{~B}$ & $10-28$ & $\begin{array}{l}\text { OR-Oak Ridge National } \\
\text { Laboratory }\end{array}$ & 1 exposed & 0 & $\begin{array}{l}\text { While work was being per- } \\
\text { formed on infrared tri- } \\
\text { tium cell, employee re- } \\
\text { ceived tritium exposure } \\
\text { of } 12.5 \text { rem to total body } \\
\text { tissue. (See page } 22 \text { ). }\end{array}$ \\
\hline $63-47 \mathrm{~B}$ & $7-1$ & RL-General Electric Co. & 0 & 7,442 & $\begin{array}{l}\text { Lightning ignited sage and } \\
\text { grass at } 18 \text { locations. }\end{array}$ \\
\hline
\end{tabular}


INDUSTRIAL ACCIDENTS AND RADIATION EXPOSURE INCIDENTS (Cont'd.)

\begin{tabular}{|c|c|c|c|c|c|}
\hline $\begin{array}{l}\text { No.* \& } \\
\text { Type } †\end{array}$ & Date & Field Office \& Contractor & $\begin{array}{c}\text { Injuriest \& } \\
\text { Exposures }\end{array}$ & $\begin{array}{l}\text { AEC Property } \\
\text { Damage }\end{array}$ & Remarks \\
\hline $63-48 \mathrm{~A}$ & $11-6$ & RL-General Electric Co. & 0 & $\$ 397,000$ & $\begin{array}{l}\text { Fire (definite cause unde- } \\
\text { termined) in plutonium } \\
\text { purification facility. } \\
\text { Plutonium contamination } \\
\text { in immediate area of fire. } \\
\text { Firemen received slight } \\
\text { skin contamination, } \\
\text { readily removed. Costs } \\
\text { related directly to fire } \\
\$ 85,400 ; \text { decontamination } \\
\text { costs } \$ 251,300 ; \text { overhead } \\
\text { related to direct losses } \\
\$ 60,300 \text {. (See page } 23 \text { ). }\end{array}$ \\
\hline $63-49 \mathrm{~B}$ & $11-8$ & $\begin{array}{l}\text { SNPO-N-Los Alamos } \\
\text { Scientific Laboratory }\end{array}$ & 0 & 70,000 & $\begin{array}{l}\text { Overpressurization of hy- } \\
\text { drogen feed lines to tur- } \\
\text { bine pump resulted in } \\
\text { destruction of turbine, } \\
\text { shrapnel damaged pipes, } \\
\text { conduits, etc. }\end{array}$ \\
\hline $63-50 \mathrm{~A}$ & $11-13$ & $\begin{array}{l}\text { AL-Mason \& Hanger-Silas } \\
\text { Mason Co., Inc., San } \\
\text { Antonio, Tex. }\end{array}$ & , & 92,568 & $\begin{array}{l}\text { High explosives }(120,000 \\
\text { detonated in storage } \\
\text { igloo. }\end{array}$ \\
\hline $63-52 \mathrm{~A}$ & $11-15$ & ID-Phillips Petroleum Co. & 0 & 25,451 & $\begin{array}{l}\text { Low-level spread of pluto- } \\
\text { nium contamination from } \\
\text { glovebox. (See page } 24 \text { ). }\end{array}$ \\
\hline $63-53 \mathrm{~A}$ & $9-26$ & AL-The Zia Co. & 1 killed & 0 & $\begin{array}{l}\text { When truck backed over can } \\
\text { of paint thinner, can rup- } \\
\text { tured, spraying contents } \\
\text { on laborer's clothing. } \\
\text { Flames from dump fire } \\
\text { ignited his coveralls, and } \\
\text { he died about six weeks } \\
\text { later as result of chemi- } \\
\text { cal burns received. (See } \\
\text { page } 42 \text { ). }\end{array}$ \\
\hline $63-54 \mathrm{~B}$ & $12-4$ & AL-Eagle-Picher Co. & 1 injured & 58,507 & $\begin{array}{l}\text { Flash fire (definite cause } \\
\text { undetermined) in vault } \\
\text { where heat-pad paper } \\
\text { stored. One employee } \\
\text { received first and sec- } \\
\text { ond degree burns. }\end{array}$ \\
\hline $63-55 \mathrm{~B}$ & $12-9$ & $\begin{array}{l}\text { OR-Goodyear Atomic } \\
\text { Corp. }\end{array}$ & 0 & 51,500 & $\begin{array}{l}\text { Compressor in cell de- } \\
\text { bladed while on-stream, } \\
\text { accompanied by small } \\
\text { fire on exterior of com- } \\
\text { pressor shell. }\end{array}$ \\
\hline $63-56 \mathrm{~A}$ & $12-12$ & ID-Phillips Petroleum Co. & 0 & 0 & $\begin{array}{l}\text { Truck transporting radio- } \\
\text { active material jack- } \\
\text { knifed. Shipment undam- } \\
\text { aged. }\end{array}$ \\
\hline $63-57 \mathrm{~B}$ & $12-11$ & RL-Government & 0 & 23,500 & $\begin{array}{l}\text { Fire, probably caused by } \\
\text { cigarette, in Government- } \\
\text { owned drug store. }\end{array}$ \\
\hline $63-59 \mathrm{~B}$ & $12-13$ & $\begin{array}{l}\text { OR-Goodyear Atomic } \\
\text { Corp. }\end{array}$ & 0 & 244,800 & $\begin{array}{l}\text { Power transformer failed } \\
\text { causing tank to rupture. } \\
\text { Insulating oil ignited im- } \\
\text { mediately. }\end{array}$ \\
\hline $63-60 \mathrm{~B}$ & $10-17$ & $\begin{array}{l}\text { CH-Argonne National } \\
\text { Laboratory }\end{array}$ & 1 exposed & 0 & $\begin{array}{l}\text { While making adjustment } \\
\text { on gradient synchrotron, } \\
\text { an employee received } \\
\text { whole-body exposure of } \\
3.8 \text { rem. (See page } 22 \text { ). }\end{array}$ \\
\hline
\end{tabular}


INDUSTRIAL ACCIDENTS AND RADIATION EXPOSURE INCIDENTS (Cont'd.)

\begin{tabular}{|c|c|c|c|c|c|}
\hline $\begin{array}{l}\text { No.* \& } \\
\text { Type } \dagger\end{array}$ & Date & Field Office \& Contractor & $\begin{array}{c}\text { Injuries } \$ \text { \& } \\
\text { Exposures }\end{array}$ & $\begin{array}{c}\text { AEC Property } \\
\text { Damage }\end{array}$ & Remarks \\
\hline $63-61 \mathrm{~B}$ & $12-21$ & $\begin{array}{l}\text { AL-Monsanto Chemical } \\
\text { Co. }\end{array}$ & 0 & $\$ 7,611$ & $\begin{array}{l}\text { During installation of cop- } \\
\text { per tubing, plastic bag } \\
\text { containing plutonium } \\
\text { oxide was punctured, re- } \\
\text { sulting in contamination } \\
\text { of the area. Cost due to } \\
\text { decontamination. (See } \\
\text { page 24). }\end{array}$ \\
\hline $63-62 \mathrm{~B}$ & $\begin{array}{l}\text { Oct }- \\
\text { Dec. }\end{array}$ & $\begin{array}{l}\text { AL-Los Alamos Scientific } \\
\text { Laboratory }\end{array}$ & 2 exposed & 0 & $\begin{array}{l}\text { Two men engaged in casting } \\
\text { operations with normal } \\
\text { uranium received whole- } \\
\text { body skin doses of } 15.17 \\
\text { rem and } 17.29 \text { rem, re- } \\
\text { spectively. (See page } 23 \text { ). }\end{array}$ \\
\hline \multirow[t]{2}{*}{$63-63 \mathrm{~B}$} & $10-31$ & $\begin{array}{l}\text { SNPO-N-Pan American } \\
\text { World Airways }\end{array}$ & 0 & 5,883 & $\begin{array}{l}\text { While in the process of } \\
\text { loading collimator onto } \\
\text { truck, crane began to tip. } \\
\text { Operator dropped load } \\
\text { onto truck in attempt to } \\
\text { save crane. Damage to } \\
\text { crane } \$ 5,483 \text {; damage to } \\
\text { truck } \$ 400 \text {. }\end{array}$ \\
\hline & 1964 & & & & \\
\hline $64-1 \mathrm{~A}$ & $1-20$ & AL-Government & 0 & 0 & $\begin{array}{l}\text { Empty AEC-owned 4-ton } \\
\text { truck skidded on icy road, } \\
\text { colliding with privately } \\
\text { owned car carrying five } \\
\text { passengers, three of } \\
\text { whom were injured. The } \\
\text { three truck couriers were } \\
\text { bruised but otherwise un- } \\
\text { injured. }\end{array}$ \\
\hline $64-2 \mathrm{~A}$ & $1-23$ & RL-General Electric Co. & 0 & 2,141 & $\begin{array}{l}\text { Several employees received } \\
\text { minor contamination } \\
\text { while replacing equip- } \\
\text { ment in waste storage } \\
\text { facilities. They were } \\
\text { readily decontaminated. } \\
\text { Cost due to decontamina- } \\
\text { tion of equipment. (See } \\
\text { page } 25 \text { ). }\end{array}$ \\
\hline $64-3 \mathrm{~A}$ & $2-3$ & $\begin{array}{l}\text { OR-Union Carbide Corp. } \\
\text { Nuclear Div. }\end{array}$ & 0 & 0 & $\begin{array}{l}\text { Interplant truck shipment } \\
\text { of radioactive materials } \\
\text { in accident with private } \\
\text { car. Shipment undam- } \\
\text { aged. }\end{array}$ \\
\hline $64-4 \mathrm{~B}$ & $1-25$ & AL-The Bendix Corp. & 0 & 5,838 & $\begin{array}{l}\text { Fire in air-conditioning } \\
\text { system, believed to have } \\
\text { been started by cutting } \\
\text { torch. }\end{array}$ \\
\hline $64-5 B$ & $2-25$ & $\begin{array}{l}\text { AL-Mason \& Hanger- } \\
\text { Silas Mason Co. } \\
\text { Burlington, Iowa }\end{array}$ & 0 & 60,400 & $\begin{array}{l}\text { Explosion (definite cause } \\
\text { undetermined) during } \\
\text { normal pressing opera- } \\
\text { tions of high explosives. } \\
\text { No radioactive material } \\
\text { involved. }\end{array}$ \\
\hline $64-6 \mathrm{~A}$ & $2-24$ & $\begin{array}{l}\text { AL-Mason \& Hanger- } \\
\text { Silas Mason Co. } \\
\text { Clarksville, Tenn. }\end{array}$ & 0 & 0 & $\begin{array}{l}\text { During routine disposal of } \\
\text { high explosives waste, } \\
\text { brush fire resulted, burn- } \\
\text { ing about } 20 \text { acres of no } \\
\text { value. No radioactive } \\
\text { material involved. }\end{array}$ \\
\hline
\end{tabular}


INDUSTRIAL ACCIDENTS AND RADIATION EXPOSURE INCIDENTS (Cont'd.)

\begin{tabular}{|c|c|c|c|c|c|}
\hline $\begin{array}{l}\text { No.* \& } \\
\text { Type } \dagger\end{array}$ & Date & Field Office \& Contractor & $\begin{array}{l}\text { Injuriest \& } \\
\text { Exposures }\end{array}$ & $\begin{array}{l}\text { AEC Property } \\
\text { Damage }\end{array}$ & Remarks \\
\hline $64-7 \mathrm{~A}$ & $2-24$ & ID-Phillips Petroleum Co. & 0 & $\$$ & $\begin{array}{l}\text { Truck carrying } 1 / 2 \text {-ton } \\
\text { shipping cask containing } \\
\text { small quantity of radio- } \\
\text { active material in colli- } \\
\text { sion. Shipment undam- } \\
\text { aged. }\end{array}$ \\
\hline $64-8 \mathrm{~B}$ & $2-4$ & NV-Holmes \& Narver, Inc. & 0 & 14,000 & $\begin{array}{l}\text { Fire (definite cause unde- } \\
\text { termined) in construction } \\
\text { supplies in storage yard. }\end{array}$ \\
\hline $64-9 \mathrm{~A}$ & $3-11$ & $\begin{array}{l}\text { SAN-Lawrence Radiation } \\
\text { Laboratory }\end{array}$ & 1 killed & 1,600 & $\begin{array}{l}\text { Electronics coordinator } \\
\text { died as result of skull } \\
\text { fracture received when } \\
\text { car which he was driving } \\
\text { overturned when he failed } \\
\text { to manipulate a curve. } \\
\text { (See page } 43 \text { ). }\end{array}$ \\
\hline $64-10 \mathrm{~B}$ & $2-12$ & RL-General Electric Co. & 0 & 14,400 & $\begin{array}{l}\text { Pressurizer vessel of re- } \\
\text { actor loop damaged as } \\
\text { result of operating heat- } \\
\text { ers without water in sys- } \\
\text { tem. }\end{array}$ \\
\hline $64-11 \mathrm{~B}$ & $3-11$ & RL-General Electric Co. & 1 exposed & 0 & $\begin{array}{l}\text { When piece of irradiated } \\
\text { wire pierced rubber glove } \\
\text { of millwright and lay } \\
\text { against his skin approxi- } \\
\text { mately three minutes, he } \\
\text { received radiation expo- } \\
\text { sure to his right thumb of } \\
57 \text { rem. (See page 27). }\end{array}$ \\
\hline $64-12 \mathrm{~A}$ & $3-15$ & $\begin{array}{l}\text { NY-Materials \& Equip- } \\
\text { ment Corp. }\end{array}$ & 0 & 0 & $\begin{array}{l}\text { Truck carrying radioactive } \\
\text { materials slid into ditch. } \\
\text { Shipment undamaged. }\end{array}$ \\
\hline $64-13 \mathrm{~B}$ & $3-19$ & CH-Atomics International & 0 & 6,075 & $\begin{array}{l}\text { Clean water, being used to } \\
\text { test two new waste tanks, } \\
\text { was contaminated by con- } \\
\text { densation from contami- } \\
\text { nated vent line connected } \\
\text { to one tank. Water subse- } \\
\text { quently drained onto } \\
\text { asphalt surface, con- } \\
\text { taminating it and drain- } \\
\text { age ditch. (See page } 28 \text { ). }\end{array}$ \\
\hline $64-14 \mathrm{~B}$ & $\begin{array}{l}\text { Jan. }_{*}- \\
\text { Mar. }\end{array}$ & $\begin{array}{l}\text { CH-Battelle Memorial } \\
\text { Institute }\end{array}$ & 1 exposed & 0 & $\begin{array}{l}\text { During routine handling of } \\
\text { fission products in hot } \\
\text { cell, technician received } \\
\text { quarterly external whole- } \\
\text { body gamma exposure of } \\
3.25 \text { rem. (See page 25). }\end{array}$ \\
\hline $64-15$ B & $4-21$ & $\begin{array}{l}\text { NV-Reynolds Electrical } \\
\quad \text { \& Engineering Co., Inc. }\end{array}$ & 1 killed & 0 & $\begin{array}{l}\text { Driver of } 8,000 \text {-gal. water } \\
\text { tanker tried to jump clear } \\
\text { of tanker when he lost } \\
\text { control of it; however, he } \\
\text { was unable to do so, and it } \\
\text { rolled over him, crushing } \\
\text { him to death. (See page } \\
44) \text {. }\end{array}$ \\
\hline $64-1.6 \mathrm{~B}$ & $4-19$ & AL-The Bendix Corp. & 0 & 5,143 & $\begin{array}{l}\text { Lightning damaged switch- } \\
\text { gear. }\end{array}$ \\
\hline $64-17 \mathrm{~B}$ & $3-31$ & CH-Atomics International & 0 & 23,000 & $\begin{array}{l}\text { Leak developed in sodium } \\
\text { loop tubing; resultant } \\
\text { vapors corroded emis- } \\
\text { sivity coating of six } \\
\text { banks of thermoelectric } \\
\text { modules, rendering them } \\
\text { unfit for further use. }\end{array}$ \\
\hline
\end{tabular}


INDUSTRIAL ACCIDENTS AND RADIATION EXPOSURE INCIDENTS (Cont'd.)

\begin{tabular}{|c|c|c|c|c|c|}
\hline $\begin{array}{l}\text { No.* \& } \\
\text { Type } \dagger\end{array}$ & Date & Field Office \& Contractor & $\begin{array}{r}\text { Injuries } \& \text { \& } \\
\text { Exposures }\end{array}$ & $\begin{array}{l}\text { AEC Property } \\
\text { Damage }\end{array}$ & Remarks \\
\hline $64-18 \mathrm{~A}$ & $4-22$ & $\begin{array}{l}\text { SAN-Advanced Technology } \\
\text { Laboratories }\end{array}$ & 0 & $\$$ & $\begin{array}{l}\text { One drum of shipment of } \\
\text { five, each drum contain- } \\
\text { ing five polyethylene bot- } \\
\text { tles of radioactive ma- } \\
\text { terials, was damaged } \\
\text { during transit. No con- } \\
\text { tamination nor release of } \\
\text { material resulted. (See } \\
\text { page } 28 \text { ). }\end{array}$ \\
\hline $64-19 \mathrm{~B}$ & $5-10$ & ID-Phillips Petroleum Co. & 0 & 12,884 & $\begin{array}{l}\text { During steam flushing to } \\
\text { remove radioactive con- } \\
\text { tamination from pipelines } \\
\text { to permit tie-in to new } \\
\text { lines, leak developed in } \\
\text { hose coupling. Contami- } \\
\text { nated fluid and steam is- } \\
\text { suing from leak were } \\
\text { rapidly dispersed by high } \\
\text { wind over approximately } \\
\text { ten acres. Majority of } \\
\text { cost due to cleanup. (See } \\
\text { page } 30 \text { ). }\end{array}$ \\
\hline $64-20 \mathrm{~B}$ & $2-15$ & $\begin{array}{l}\text { SR-E. I. du Pont } \\
\text { de Nemours \& Co. }\end{array}$ & 0 & 10,460 & $\begin{array}{l}\text { Plywood block left in re- } \\
\text { actor pipeline system } \\
\text { following repairs disinte- } \\
\text { grated causing nonradio- } \\
\text { active contamination of } \\
\text { line. (See page } 26 \text { ). }\end{array}$ \\
\hline $64-21 \mathrm{~B}$ & $\begin{array}{l}\text { Jan.- } \\
\text { Mar. }\end{array}$ & PNR-Duquesne Light Co. & 2 exposed & 0 & $\begin{array}{l}\text { Due to error in adding } \\
\text { doses, two employees re- } \\
\text { ceived whole-body dose } \\
\text { of } 3.26 \text { rem and } 3.12 \text { rem, } \\
\text { respectively. (See page } \\
26) \text {. }\end{array}$ \\
\hline $64-22 \mathrm{~B}$ & $4-23$ & $\begin{array}{l}\text { SR-E. I. du Pont } \\
\text { de Nemours \& Co. }\end{array}$ & 0 & & $\begin{array}{l}\text { Tritium release to atmo- } \\
\text { sphere through stack. } \\
\text { (See page } 29 \text { ). }\end{array}$ \\
\hline $64-23 \mathrm{~A}$ & $5-18$ & $\begin{array}{l}\mathrm{CH} \text {-Battelle Memorial } \\
\text { Institute }\end{array}$ & 0 & 0 & $\begin{array}{l}\text { Cask, being shipped by } \\
\text { truck, found at destina- } \\
\text { tion to be emitting } 6 \\
\text { rem/hr (gamma). In- } \\
\text { vestigation revealed cask } \\
\text { had been dropped en route. } \\
\text { Slight area contamina- } \\
\text { tion; no significant } \\
\text { personnel radiation expo- } \\
\text { sures. (See page } 31 \text { ). }\end{array}$ \\
\hline $64-24 \mathrm{~B}$ & $7-27$ & $\begin{array}{l}\text { NY-Westinghouse Electric } \\
\text { Corp. }\end{array}$ & 0 & 10,500 & $\begin{array}{l}\text { When a junction box de- } \\
\text { veloped a short or ground, } \\
\text { sparks ignited wooden } \\
\text { crates nearby. }\end{array}$ \\
\hline $64-25 B$ & $6-12$ & AL-Dow Chemical Co. & 1 exposed & 56,400 & $\begin{array}{l}\text { Chemical explosion caused } \\
\text { by burning plutonium } \\
\text { chips accidentally } \\
\text { dropped into carbon } \\
\text { tetrachloride bath. The } \\
\text { thumb and index finger of } \\
\text { the operator had to be } \\
\text { amputated to effect decon- } \\
\text { tamination because flying } \\
\text { fragments of plutonium } \\
\text { had become embedded in } \\
\text { them. (See page } 32 \text { ). }\end{array}$ \\
\hline
\end{tabular}


INDUSTRIAL ACCIDENTS AND RADIATION EXPOSURE INCIDENTS (Cont'd.)

\begin{tabular}{|c|c|c|c|c|c|}
\hline $\begin{array}{c}\text { No.* \& } \\
\text { Typet }\end{array}$ & Date & Freld Offlce \& Contractor & $\begin{array}{r}\text { Injuries } \& \\
\text { Exposures }\end{array}$ & $\begin{array}{c}\text { AEC Property } \\
\text { Damage }\end{array}$ & Remarks \\
\hline $64-26 \mathrm{~A}$ & $5-26$ & $\begin{array}{l}\text { OR-Mallinckrodt Chemical } \\
\text { Works }\end{array}$ & 0 & $\$$ & $\begin{array}{l}\text { Tractor-trailer hauling ra- } \\
\text { dioactive material involved } \\
\text { in accident with private } \\
\text { car. Shipment undamaged. }\end{array}$ \\
\hline $64-27 \mathrm{~B}$ & $6-10$ & $\begin{array}{l}\text { NV-Reynolds Electrical } \\
\quad \text { \& Engineering Co., Inc. }\end{array}$ & 0 & 5,000 & $\begin{array}{l}\text { Vacuum unit, mounted on } \\
\text { semitrailer, being towed } \\
\text { by flat-bed truck, which } \\
\text { jackknifed, causing semi- } \\
\text { trailer to turn over, } \\
\text { damaging vacuum unit. }\end{array}$ \\
\hline $64-28 \mathrm{~B}$ & $6-3$ & $\begin{array}{l}\text { SR-E. I. du Pont } \\
\text { de Nemours \& Co. }\end{array}$ & 0 & & $\begin{array}{l}\text { Tritium release to atmo- } \\
\text { sphere through stack. } \\
\text { (See page } 31 \text { ). }\end{array}$ \\
\hline $64-29 \mathrm{~B}$ & $6-14$ & $\begin{array}{l}\text { SR-L. I. du Pont } \\
\text { de Nemours \& Co. }\end{array}$ & 0 & 8,400 & $\begin{array}{l}\text { Loss of approximately } 600 \\
\text { lbs. of heavy water } \\
\text { through pipe left open } \\
\text { inadvertently. }\end{array}$ \\
\hline $64-30 \mathrm{~B}$ & $6-18$ & $\begin{array}{l}\text { CH-Notre Dame Un1- } \\
\text { versity }\end{array}$ & 2 exposed & 0 & $\begin{array}{l}\text { Exposures of approximately } \\
180 \text { rem to the fingers } \\
\text { suffered by two em- } \\
\text { ployees while working } \\
\text { w1th } 2000 \text {-curie cobalt } 60 \\
\text { source. (See page } 32 \text { ). }\end{array}$ \\
\hline $64-31 \mathrm{~A}$ & $7-1$ & $\begin{array}{l}\text { A L-Federal Roofing \& } \\
\text { Siding Co. }\end{array}$ & 1 killed & 0 & $\begin{array}{l}\text { Roofer died three days } \\
\text { later from results of } \\
\text { head and internal in- } \\
\text { juries sustained when he } \\
\text { fell } 22 \text { ' to the ground } \\
\text { from the roof where he } \\
\text { was working. (See page } \\
44 \text { ). }\end{array}$ \\
\hline $64-32 \mathrm{~B}$ & $7-2$ & $\begin{array}{l}\text { AL-Los Alamos Scientific } \\
\text { Laboratory }\end{array}$ & 0 & 11,597 & $\begin{array}{l}\text { Explosion, attributed to ac- } \\
\text { cumulation of unburned } \\
\text { gas in area of stagnant } \\
\text { air movement in furnace } \\
\text { upper passageways, dam- } \\
\text { aged boller furnace. }\end{array}$ \\
\hline $64-33 \mathrm{~B}$ & $6-28$ & $\begin{array}{l}\text { SR-E. I du Pont } \\
\text { de Nemours \& Co }\end{array}$ & 0 & & $\begin{array}{l}\text { Tritium release to atmo- } \\
\text { sphere through stack. } \\
\text { (See page } 33 \text { ). }\end{array}$ \\
\hline $64-34 \mathrm{~B}$ & $6-19$ & $\begin{array}{l}\text { SR-E. I. du Pont } \\
\text { de Nemours \& Co. }\end{array}$ & 0 & & $\begin{array}{l}\text { Tritium release to atmo- } \\
\text { sphere through stack. } \\
\text { (See page } 33 \text { ). }\end{array}$ \\
\hline $64-35 \mathrm{~A}$ & $7-10$ & $\begin{array}{l}\text { OR-National Lead Co, of } \\
\text { Ohio }\end{array}$ & 0 & 0 & $\begin{array}{l}\text { Drum of radioactive mate- } \\
\text { rial and trailer transport- } \\
\text { ing it slightly contami- } \\
\text { nated. Both readily } \\
\text { decontaminated. No ma- } \\
\text { terial release nor radia- } \\
\text { tion exposures. (See } \\
\text { page } 33 \text { ) }\end{array}$ \\
\hline $64-36 \mathrm{~B}$ & $\begin{array}{l}4-27 / \\
5-11\end{array}$ & $\begin{array}{l}\text { AL-Monsanto Research } \\
\text { Corp. }\end{array}$ & 1 exposed & 0 & $\begin{array}{l}\text { During routine work with } \\
\text { polonium in a laboratory, } \\
\text { a chemist received a kid- } \\
\text { ney dose of approximately } \\
45 \text { rem in ensuing year. } \\
\text { (See page 29). }\end{array}$ \\
\hline
\end{tabular}


INDUSTRIAL ACCIDENTS AND RADIATION EXPOSURE INCIDENTS (Cont'd.)

\begin{tabular}{|c|c|c|c|c|c|}
\hline $\begin{array}{l}\text { No.* \& } \\
\text { Typef }\end{array}$ & Date & Field Office \& Contractor & $\begin{array}{r}\text { Injuries }+8 \\
\text { Exposures }\end{array}$ & $\begin{array}{l}\text { AEC Property } \\
\text { Damage }\end{array}$ & Remarks \\
\hline $64-37 \mathrm{~B}$ & $7-10$ & RL-General Electric Co. & 1 exposed & 750. & $\begin{array}{l}\text { During pressing operations } \\
\text { within glovebox, pluto- } \\
\text { nium sample shattered. } \\
\text { Ejected fragments caused } \\
\text { deep laceration of em- } \\
\text { ployee's arm and minor } \\
\text { contamination of adjacent } \\
\text { working area. (See page } \\
\text { 34). }\end{array}$ \\
\hline $64-38 A$ & $7-23$ & $\begin{array}{l}\text { OR-National Lead Co. of } \\
\text { Ohio }\end{array}$ & 0 & 0 & $\begin{array}{l}\text { When door of freight car } \\
\text { broke open during switch- } \\
\text { ing operations, two drums } \\
\text { containing radioactive } \\
\text { material fell out. Part of } \\
\text { contents of one drum } \\
\text { spilled in yard area but } \\
\text { was recovered. (See } \\
\text { page } 35 \text { ). }\end{array}$ \\
\hline $64-39 \mathrm{~B}$ & $4-17$ & RL-General Electric Co. & 0 & 51,300 & $\begin{array}{l}\text { Charging exror made in } \\
\text { loading reactor resulted } \\
\text { in exceeding operating } \\
\text { limits. (See page } 28 \text { ). }\end{array}$ \\
\hline $64-40 \mathrm{~B}$ & $7-22$ & ID-Phillips Petroleum Co. & 1 exposed & 0 & $\begin{array}{l}\text { Employee received expo- } \\
\text { sure of approximately } 31 \\
\text { rem to one hand and } 5 \\
\text { rem to the other while } \\
\text { manually removing ra- } \\
\text { dioactive flux wires from } \\
\text { stainless steel tubes. } \\
\text { (See page } 34 \text { ). }\end{array}$ \\
\hline $64-41 \mathrm{~B}$ & $7-21$ & $\begin{array}{l}\text { BH-Brookhaven National } \\
\text { Laboratory }\end{array}$ & 0 & 11,613 & $\begin{array}{l}\text { Explosion in hydrogen puri- } \\
\text { fier in bubble changer } \\
\text { expansion system when } \\
\text { valve inadvertently left in } \\
\text { closed position during } \\
\text { purging operation. Pre- } \\
\text { cooler and adsorber coils } \\
\text { torn open and containing } \\
\text { dewar bulged. }\end{array}$ \\
\hline $64-42 \mathrm{~B}$ & $8-13$ & $\begin{array}{l}\text { NV-Reynolds Electrical } \\
\quad \text { \& Engineering Co., Inc. }\end{array}$ & 0 & 10,000 & $\begin{array}{l}\text { Mobile drill rig developed } \\
\text { excessive speed on down- } \\
\text { grade, failed to negotiate } \\
\text { curve, and turned over, } \\
\text { damaging rig carriage. }\end{array}$ \\
\hline $64-43 B$ & $\begin{array}{l}7-17 / \\
7-30\end{array}$ & $\begin{array}{l}\text { SR-E. I. du Pont } \\
\text { de Nemours \& Co. }\end{array}$ & 0 & 6,000 & $\begin{array}{l}\text { Leaking nitric acid cor- } \\
\text { roded canyon cell equip- } \\
\text { ment beyond repair. }\end{array}$ \\
\hline $64-44 \mathrm{~A}$ & $8-22$ & $\begin{array}{l}\text { SR-Sylcor Division of } \\
\text { Sylvania Electric } \\
\text { Products }\end{array}$ & 0 & 2,300 & $\begin{array}{l}\text { Trailer-truck loaded with } \\
\text { radioactive material col- } \\
\text { lided with private car. } \\
\text { Loss represents direct } \\
\text { damage to fuel elements. } \\
\text { Four non-AEC persons } \\
\text { injured. }\end{array}$ \\
\hline $64-45 \mathrm{~B}$ & $9-2$ & RL-General Electric Co. & 0 & 8,800 & $\begin{array}{l}\text { During normal operations, } \\
\text { plastic impeller of ex- } \\
\text { haust fan disintegrated. } \\
\text { Fragments penetrated } \\
\text { housing of adjacent fan } \\
\text { causing it to disintegrate. } \\
\text { Nearby water line was } \\
\text { broken and a third fan } \\
\text { unit damaged by the fly- } \\
\text { ing pieces. }\end{array}$ \\
\hline
\end{tabular}


INDUSTRIAL ACCIDENTS AND RADIATION EXPOSURE INCIDENTS (Cont'd.)

\begin{tabular}{|c|c|c|c|c|c|}
\hline $\begin{array}{c}\text { No.* \& } \\
\text { Type } †\end{array}$ & Date & Field Office \& Contractor & $\begin{array}{r}\text { Injuries: \& } \\
\text { Exposures }\end{array}$ & $\begin{array}{l}\text { AEC Property } \\
\text { Damage }\end{array}$ & Remarks \\
\hline $64-46 \mathrm{~B}$ & $\begin{array}{l}\text { Apr.- } \\
\text { June }\end{array}$ & $\begin{array}{l}\text { SAN-Lawrence Radiation } \\
\text { Laboratory }\end{array}$ & 1 exposed & 0 & $\begin{array}{l}\text { Chemist working in vicinity } \\
\text { of cyclotron received } \\
\text { whole-body dose of } 3.4 \\
\text { rem: (See page } 30 \text { ). }\end{array}$ \\
\hline $64-47 \mathrm{~A}$ & $9-2$ & $\begin{array}{l}\mathrm{CH} \text {-Battelle Memorial } \\
\text { Institute }\end{array}$ & 0 & 0 & $\begin{array}{l}\text { Truck-trailer arrived at } \\
\text { destination with trailer } \\
\text { bed slightly contaminated } \\
\text { by gross fission products } \\
\text { coming from surface of } \\
\text { cask containing irradi- } \\
\text { ated material. Truck- } \\
\text { trailer decontaminated } \\
\text { and returned to carrier. } \\
\text { (See page } 35 \text { ). }\end{array}$ \\
\hline $64-48$ B & $9-14$ & $\begin{array}{l}\text { NV-Reynolds Electrical } \\
\quad \text { \& Engineering Co., Inc. }\end{array}$ & 0 & 12,000 & $\begin{array}{l}\text { Defective weld on drill rig } \\
\text { leg caused leg to buckle } \\
\text { inward and the mast to } \\
\text { fall. }\end{array}$ \\
\hline $64-49$ A & $9-19$ & $\begin{array}{l}\text { NV-Reynolds Electrical } \\
\quad \text { \& Engineering Co., Inc. }\end{array}$ & $\begin{array}{l}1 \text { killed } \\
1 \text { injured }\end{array}$ & 74,000 & $\begin{array}{l}\text { Electrical cables were be- } \\
\text { ing lowered into under- } \\
\text { ground complex when a } \\
\text { wire rope from a hoist } \\
\text { broke causing cable } \\
\text { spools to be jerked from } \\
\text { their racks. Four men } \\
\text { were hit by the spools, } \\
\text { one receiving extensive } \\
\text { head and chest injuries } \\
\text { from which he died, one } \\
\text { being seriously injured, } \\
\text { other two receiving lesser } \\
\text { injuries. Four men were } \\
\text { trapped underground but } \\
\text { were rescued. (See page } \\
\text { 44). }\end{array}$ \\
\hline $64-50 \mathrm{~A}$ & $9-23$ & SAN-Case Foundation Co. & 1 killed & 0 & $\begin{array}{l}\text { Employee electrocuted } \\
\text { when in contact with drill } \\
\text { rig which struck overhead } \\
\text { power lines. (See page 45) }\end{array}$ \\
\hline $64-51 \mathrm{~B}$ & $\begin{array}{r}1-22 / \\
3-2\end{array}$ & RL-Peter Kiewit Sons' Co. & 0 & 66,000 & $\begin{array}{l}1500 \text { feet of disposal tunnel } \\
\text { collapsed while back- } \\
\text { filling operations were in } \\
\text { progress. Six weeks } \\
\text { later, another } 50 \text { feet } \\
\text { collapsed. }\end{array}$ \\
\hline $64-52 \mathrm{~B}$ & $7-13$ & $\begin{array}{l}\text { SAN-Lawrence Radiation } \\
\text { Laboratory }\end{array}$ & 0 & 9,500 & $\begin{array}{l}\text { High explosives accidentally } \\
\text { exploded and burned dur- } \\
\text { ing normal pressing op- } \\
\text { eration. No radioactive } \\
\text { material involved. }\end{array}$ \\
\hline $64-53 \mathrm{~B}$ & $7-24$ & $\begin{array}{l}\text { SR-E. I. du Pont } \\
\text { de Nemours \& Co. }\end{array}$ & 0 & & $\begin{array}{l}\text { Tritium release to atmo- } \\
\text { sphere through stack. } \\
\text { (See page } 35 \text { ). }\end{array}$ \\
\hline $64-54 \mathrm{~B}$ & $10-1$ & $\begin{array}{l}\text { SR-E. I. du Pont } \\
\text { de Nemours \& Co. }\end{array}$ & 0 & 21,000 & $\begin{array}{l}\text { Fire (definite cause unde- } \\
\text { termined) occurred } \\
\text { around anion exchange } \\
\text { column in hot canyon. } \\
\text { Fire caused airborne } \\
\text { contamination to crane } \\
\text { used for remote main- } \\
\text { tenance. Water to quench } \\
\text { fire damaged electric } \\
\text { motors. (See page } 36 \text { ). }\end{array}$ \\
\hline
\end{tabular}


INDUSTRIAL ACCIDENTS AND RADIATION EXPOSURE INCIDENTS (Cont'd.)

\begin{tabular}{|c|c|c|c|c|c|}
\hline $\begin{array}{l}\text { No.* \& } \\
\text { Type }\end{array}$ & Date & Field Office \& Contractor & $\begin{array}{r}\text { Injuriest \& } \\
\text { Exposures }\end{array}$ & $\begin{array}{l}\text { AEC Property } \\
\text { Damage }\end{array}$ & Remarks \\
\hline $64-55 \mathrm{~B}$ & $10-27$ & $\begin{array}{l}\text { AL-Monsanto Research } \\
\text { Corp. }\end{array}$ & 0 & $\$ 34,922$ & $\begin{array}{l}\text { Chemical explosion in } \\
\text { metal hood when methanol } \\
\text { vapors reached flash- } \\
\text { point. Two sets of gloves } \\
\text { were shredded by the ex- } \\
\text { plosion. Contamination } \\
\text { spread in operating area. } \\
\text { Three employees received } \\
\text { slight contamination. (See } \\
\text { page 36). }\end{array}$ \\
\hline $64-56 \mathrm{~A}$ & $11-3$ & RL-General Electric Co. & 0 & 316,900 & $\begin{array}{l}\text { Roof fire (definite cause } \\
\text { undetermined) resulted } \\
\text { in extensive damage to } \\
\text { biology building. All lab- } \\
\text { oratory experiments and } \\
\text { fish housed in building } \\
\text { lost. Also some valuable } \\
\text { records. }\end{array}$ \\
\hline $64-57 \mathrm{~A}$ & $11-11$ & $\begin{array}{l}\text { CH-Argonne National } \\
\text { Laboratory }\end{array}$ & 0 & 0 & $\begin{array}{l}\text { Broken valve on autoclave, } \\
\text { housed in lead shipping } \\
\text { cask, allowed contami- } \\
\text { nated water to seep out } \\
\text { of cask during transit, } \\
\text { contaminating container } \\
\text { and truck floor. (See } \\
\text { page } 37 \text { ). }\end{array}$ \\
\hline $64-59 \mathrm{~B}$ & $11-16$ & $\begin{array}{l}\text { OR-Union Carbide Corp., } \\
\text { Nuclear Div. }\end{array}$ & 0 & 16,000 & $\begin{array}{l}\text { Explosion in first stage of } \\
\text { nitrogen compressor re- } \\
\text { sulting in shrapnel being } \\
\text { thrown through roof and } \\
\text { also damaging sides of } \\
\text { building in which com- } \\
\text { pressor was housed. }\end{array}$ \\
\hline $64-60 \mathrm{~B}$ & $\begin{array}{l}\text { Feb.- } \\
\text { Mar. }\end{array}$ & $\begin{array}{l}\text { SAN-Lawrence Radiation } \\
\text { Laboratory }\end{array}$ & 1 exposed & 0 & $\begin{array}{l}\text { During routine laboratory } \\
\text { work, employee received } \\
\text { quarterly exposure of } 8.2 \\
\text { rem whole body. (See } \\
\text { page } 27 \text { ). }\end{array}$ \\
\hline $64-61 \mathrm{~B}$ & $11-27$ & OR-General Electric Co. & 0 & 41,680 & $\begin{array}{l}\text { Fire in } 10,000 \mathrm{KVA} \text { trans- } \\
\text { former. }\end{array}$ \\
\hline $64-63 \mathrm{~A}$ & $12-15$ & $\begin{array}{l}\text { NV-Reynolds Electrical } \\
\text { \& Engineering Co., Inc. }\end{array}$ & $\begin{array}{l}1 \text { killed } \\
1 \text { injured }\end{array}$ & 2,340 & $\begin{array}{l}\text { When drill rig assembly } \\
\text { failed, swivel assembly } \\
\text { pipe fell. Rig operator } \\
\text { killed instantly from } \\
\text { crushing blow on head. } \\
\text { Another employee re- } \\
\text { ceived head injuries and } \\
\text { broken arm. (See page } \\
\text { 46). }\end{array}$ \\
\hline $64-64 \mathrm{~A}$ & $12-8$ & RL-General Electric Co. & $\begin{array}{l}1 \text { killed } \\
1 \text { injured }\end{array}$ & 504 & $\begin{array}{l}\text { Head-on collision of inter- } \\
\text { plant locomotive and } \\
\text { maintenance car. One } \\
\text { employee riding in main- } \\
\text { tenance car died two } \\
\text { weeks later from injuries } \\
\text { received; second employee } \\
\text { seriously injured. Cost } \\
\text { due to repair of main- } \\
\text { tenance car. (See page } \\
\text { 45). }\end{array}$ \\
\hline $64-65 \mathrm{~B}$ & $12-22$ & ID-Phillips Petroleum Co. & 0 & 10,000 & $\begin{array}{l}\text { High winds damaged } \\
\text { movable test cell build- } \\
\text { ing. }\end{array}$ \\
\hline $64-66 \mathrm{~B}$ & $12-22$ & $\begin{array}{l}\text { SR-E. I. du Pont } \\
\text { de Nemours \& Co. }\end{array}$ & 0 & & $\begin{array}{l}\text { Tritium release to atmo- } \\
\text { sphere through stack. } \\
\text { (See page } 37 \text { ). }\end{array}$ \\
\hline
\end{tabular}




\begin{tabular}{|c|c|c|c|c|c|}
\hline $\begin{array}{l}\text { No.* \& } \\
\text { Type } †\end{array}$ & Date & Field Office \& Contractor & $\begin{array}{r}\text { Injuriest \& } \\
\text { Exposures }\end{array}$ & $\begin{array}{l}\text { AEC Property } \\
\text { Damage }\end{array}$ & Remarks \\
\hline $64-67 \mathrm{~B}$ & $\begin{array}{r}10-28 \\
11-2\end{array}$ & $\begin{array}{l}\text { NV-Holmes \& Narver, } \\
\text { Inc. }\end{array}$ & 0 & $\$ 30,000$ & $\begin{array}{l}\text { Crane damaged while } \\
\text { aboard ship during high } \\
\text { seas accompanied by } \\
\text { gusty winds. }\end{array}$ \\
\hline $64-68 \mathrm{~B}$ & $12-17$ & $\begin{array}{l}\text { NV-Reynolds Electrical } \\
\text { \& Engineering Co., Inc. }\end{array}$ & 0 & 17,500 & $\begin{array}{l}\text { Failure of bridle line al- } \\
\text { lowed crane mast to fall } \\
\text { to derrick stand, causing } \\
\text { excessive bending of mast } \\
\text { members. }\end{array}$ \\
\hline $64-69 \mathrm{~B}$ & Dec. & $\begin{array}{l}\text { OR-Union Carbide Corp., } \\
\text { Nuclear Div. }\end{array}$ & 1 exposed & 0 & $\begin{array}{l}\text { While sampling, weighing, } \\
\text { and grinding uranium } \\
\text { oxides of varying assays } \\
\text { in enclosed process sys- } \\
\text { tem, operator received a } \\
\text { lung burden of } 0.046 \mu \mathrm{c} \text {. } \\
\text { The employee will re- } \\
\text { ceive an estimated inte- } \\
\text { grated lung dose of } 19.4 \\
\text { rem during the first year. } \\
\text { (See page } 38 \text { ). }\end{array}$ \\
\hline $64-70 \mathrm{~B}$ & $12-28$ & $\begin{array}{l}\text { SAN-General Atomic- } \\
\text { Division of General } \\
\text { Dynamics Corp. }\end{array}$ & 0 & 20,000 & $\begin{array}{l}\text { Five plutonium-fueled pro- } \\
\text { totype generators were } \\
\text { destroyed when malfunc- } \\
\text { tion of high temperature } \\
\text { environmental test oven } \\
\text { caused generators to be } \\
\text { subject to ambient tem- } \\
\text { perature of over } 600^{\circ} \mathrm{F} \text {. } \\
\text { Generators are designed } \\
\text { to withstand maximum } \\
\text { ambient temperature of } \\
165^{\circ} \mathrm{F} \text {. }\end{array}$ \\
\hline
\end{tabular}

*Division of Operational Safety, USAEC Headquarters, File Number.

f"Type A" and "Type B" accidents are defined below:

"Type A":

1. Loss or Damage to Government Property, $\$ 100,000-$ up

2. Injury or Death

a. Fatal or imminently fatal injury.

b. Five or more injuries in one accident.

3. Radiation Exposures

a. 25 rem or more to the whole body.

b. 150 rem or more to the skin.

c. 375 rem or more to the feet, ankles, hands and/or forearms.

d. Any release of radioactive material offsite which exceeds RPG's.

e. 25 rem or more external whole body during the calendar year.

$\mathrm{f}$. Any unplanned release of radioactive material which exceeds in 24 hours, $5000 \times$ Appendix B Table II-10CFR20.

4. Public Interest

a. Any accident or radiation exposure which gives rise to an inquiry by members of the public; or an inquiry from the press; or which the field office manager believes to have public information significance.

b. Any offsite accident involving vehicles carrying AEC shipments of radioactive materials.

5. Miscellaneous

a. Any accident in which an atomic or nuclear weapon (under the jurisdiction of AEC) is involved and where damage is inflicted to persons or private property.

b. Any injury or industrial illness following cumulative or massive exposure to internal or external ionizing radiation which might reasonably be expected to have caused the illness or injury and when so diagnosed by a physician competent in nuclear medicine.

"Type B"?

1. Loss or Damage to Government Property: $\$ 5,000$ to $\$ 99,999$

2. Radiation Exposures

a. When in one calendar quarter exposure exceeds: 3 rem or more whole body; 10 rem skin; 25 rem to feet, hands, ankles and/or forearms.

b. Any dose which exceeds $(\mathrm{N}-18) 5$ rem.

c. Internal body deposition which exceeds RPG's.

Lost-time injury as defined in ASA Z16.1,

$\$$ Tritium releases resulted in material losses only (\$ figure classified). 


\section{RADIATION EXPOSURE OF AEC CONTRACTOR PERSONNEL}

In accordance with its health and safety responsibilities, the AEC must conduct its activities in such a manner as to assure that radiation exposures of employees are reduced to the lowest practical levels within established limits. To carry out this responsibility, it has been necessary for the AEC and its contractors to monitor routinely all employees who might receive a significant radiation dose. A survey of AEC and AEC contractor employees showed that, of the more than 113,000 employees monitored in 1963 and 128,000 employees in 1964, 99.9 per cent received less than $5 \mathrm{rems}$ within the year and that 95.1 percent received only one rem or less. The following table gives details for the two years.

\begin{tabular}{ccc}
$\begin{array}{c}\text { EXPOSURE OF AEC AND AEC CONTRACTOR PERSONNEL TO } \\
\text { WHOLE-BODY PENETRATING RADIATION, SUMMARIZED } \\
\text { FOR 1963-64 }\end{array}$ & \\
$\begin{array}{c}\text { Range of annual } \\
\text { total exposure } \\
\text { in Rems* }\end{array}$ & 1964 & 1963 \\
\hline $0-1$ & No. of employees & No. of employees \\
$1-2$ & 122,711 & 107,786 \\
$2-3$ & 3,583 & 3,306 \\
$3-4$ & 1,823 & 1,625 \\
$4-5$ & 575 & 388 \\
$5-6$ & 176 & 153 \\
$6-7$ & 43 & 60 \\
$7-8$ & 20 & 10 \\
$8-9$ & 10 & 8 \\
$9-10$ & 7 & 2 \\
$10-11$ & 6 & 0 \\
$11-12$ & 10 & $1 \dagger$ \\
$12-$ plus & 1 & 113,339 \\
TOTAL & 0 & 0 \\
\hline
\end{tabular}

*The rem is a measure of the dose of any ionizing radiation to body tissues in terms of its estimated biological effect relative to a dose of one roentgen of high-voltage X-rays.

$\dagger$ See page 19 . 


\section{ACCIDENTS AND INCIDENTS IN AEC FACILITIES INVOLVING RADIOACTIVE MATERIAL 1963-1964}

\section{Leakage Of Radioactive Material During Shipment*}

Jersey City, N. J., Jan. 14, 1963-Ref: 63-5

Nature of Incident

Leakage of radioactive material (plutonium-contaminated precious metals, both solid and liquid) during shipment resulted in extensive contamination spread.

\section{Description of Operation}

Truck shipment of special nuclear material.

\section{Details of Incident}

During transit, plutonium-contaminated liquid leaked from a glass carboy (with a loosefitting ceramic stopper) and contaminated several trailers, truck terminals, a number of packages of other materials and several persons handling the materials.

The leakage was detected during a routine monitoring of the unloading platform after the shipment was unloaded at its destination.

The 13-gallon glass carboy was in a large wooden box. The box was marked "this end up," so that during transit the carboy would remain upright. However, the presence of pallets on the box apparently suggested to the carrier that the box should be placed on its side, resting on the pallets, which allowed the material to leak slowly from the container and the wooden box. (The carboy was not surrounded on all sides by an absorbent material sufficient to absorb the entire liquid contents.)

Nature of Exposure or Loss

Internal and external dosimetry data confirmed that there were no significant exposures. Decontamination was carried out successfully in the truck terminals, trailers, and on the cargo at a cost of approximately $\$ 27,500$.

*During 1963, there were six incidents involving trucks or trains transporting radioactive materials in which no radioactive material was released and there was no loss of integrity to the package. In a seventh incident, radioactive material was released, but confined to the package. (See TID 16764, Suppl, 2, for details). 


\section{Spread Of Low-Level Contamination}

Richland, Wash., Feb. 12, 1963 -Ref: $63-12$

Nature of Incident

Inadvertent spread of promethium 147 contamination.

Description of Operation

Preparing dilution sample for counting.

Details of Incident

While in storage, a lead cask (pig), was upset and returned to an upright position by an employee. The cask contained five cork-stoppered, glass vials (each vial containing $30 \mathrm{mg}$ of promethium 147 dissolved in $31 \mathrm{ml}$ of dilute perchloric acid). Although the pig was contained in a plastic bag, an examination after the incident showed several holes had been worn through the bag, possibly due to the shifting of the pig in the small storage area.

The undetected material leaked onto the floor. Later on, another employee enter ed the storage area, unaware of the contaminated floor. He stepped in the contamination and subsequently tracked it to other areas in the laboratory building. This resulted in extensive spread of the contamination by movements of laboratory personnel.

\section{Nature of Exposure or Loss}

Personnel received minor skin contamination and/or internal deposition through ingestion or inhalation. The maximum skin dose was $4.5 \mathrm{rem}$ to a spot on the hand of one employee; another received a hand exposure of $3.5 \mathrm{rem}$. Internal dosimetry data confirmed that there were no significant internal depositions of promethium 147. Decontamination cost was $\$ 8,150$

\section{Tritium Release}

Aiken, S. C., Mar. 14, 1963-Ref: 63-16

Nature of Incident

Accidental release of tritium to atmosphere through stack.

Description of Operation

Plant's separation facilities.

\section{Details of Incident}

An operator, under the assumption that he was closing the valve on a container of tritium, mistakenly loosened the process fitting. This allowed the gas in the container to escape through the loosened fitting.

\section{Nature of Exposure or Loss}

No tritium passed out of the hood into the process room; consequently, there were no personnel exposures. No area contaminations were noted. 


\section{Nuclear Excursion}

Livermore, Calif., Mar. 26, 1963 -Ref: 63-17

Nature of Incident

Nuclear excursion occurred accidentally during experiment.

Description of Operation

Conducting an experiment (remotely) in critical assembly facility.

Details of Incident

A nuclear excursion and subsequent fire took place during a subcritical experiment in a shielded vault designed for critical assembly experiments. The excursion was estimated at $4 \times 10^{17}$ fissions and was followed by oxidation of the enriched uranium metal in the assembly. The excursion is believed to have been caused by an unplanned slippage of a part of the assembly.

Nature of Exposure or Loss

Since the experiment was operated from the remote control room, there were no significant radiation exposures.

The total property loss was $\$ 94,881$.

\section{Radiation Exposure}

Canoga Park, Calif., Apr.15-May 8, 1963-Ref: 63-27

Nature of Incident

Employee exposed inadvertently to low energy radiation.

Description of Operation

Adjustment and alignment of goniometer on X-ray diffraction machine.

Details of Incident

The employee's manipulations in adjusting the goniometer placed him at a distance between $1 \frac{1}{2}$ and 2 feet from the apparatus, at which time he was exposed.

Nature of Exposure or Loss

The exposure $(11 \mathrm{rem})$ was to the midchest region only and resulted primarily in a skin dose without significant exposure to the eyes or gonads.

\section{Minor Exposure To Radioactivity}

Richland, Wash., Apr. 17, 1963-Ref: 63-20

Nature of Incident

Maintenance worker accidentally exposed to radiation. 
Description of Operation

Working on rear face of reactor removing ruptured slug.

Details of Incident

When a reactor was shut down because of indications of a fuel element jacket failure in a process tube, a maintenance craftsman working in the discharge area handled a rear steel sleeve (gun barrel), unaware that the gun barrel contained an irradiated fuel element in the tip. The gun barrel was removed in the course of maintenance activity to remove a stuck ruptured fuel element from the reactor.

Nature of Exposure or Loss

Employee received a whole-body radiation exposure of 5 rem.

\section{Spill Of Contaminated Solution}

Golden, Colo., Apr. 23, 1963-Ref: 63-31

Nature of Incident

Spill of contaminated nitric acid solution.

Description of Operation

Transferring waste from storage tank.

Details of Incident

Nitric acid overflowed onto the floor while being pumped into a tank.

Nature of Exposure or Loss

Cleanup and decontamination cost due to the spill of approximately 85 liters of contaminated nitric acid solution was $\$ 3,120$. In addition, equipment valued at $\$ 2,542$ had to be discarded.

\section{Radiation Exposures}

Cincinnati, Ohio, Apr.-June 1963-Ref: 63-38

Nature of Incident

Two employees received radiation exposures to the whole body and the hands.

Description of Operation

Making measurements of irradiated samples.

\section{Details of Incident}

Two men, working as a team, were making measurements of some of the physical proper ties of beryllium oxide samples (approximately 1,000) which had undergone reactor irradiation. Work was performed in several laboratories, depending upon the type of measurement being made; however, in every case, the workers were either shielded or isolated to prevent others 
from being exposed unknowingly. The employees were aware at all times of the radiation levels from the samples with which they were working.

Nature of Exposure or Loss

The men received the following external exposures:

$$
\text { Whole body Left hand Right hand }
$$

$\begin{array}{llll}\text { Employee "A" } & 3.9 \mathrm{rem} & 32 \mathrm{rem} & 14 \mathrm{rem} \\ \text { Employee "B" } & 2.1 \mathrm{rem} & 27 \mathrm{rem} & 25 \mathrm{rem}\end{array}$

Due to the nature of the work, the exposures were accumulated over the quarter and were not the result of a single exposure or incident.

\section{Construction Workers Exposed}

Richland, Wash., May 16, 1963 - Ref: $63-25$

Nature of Incident

Construction contractor workers exposed to radiation.

Description of Operation

Licensee performing radiography work at AEC construction site.

Details of Incident

During the process of removing control cables from a radiographic camera, a 27-curie iridium 192 sealed source fell out of the cable and remained unnoticed on the floor of the cell for approximately five hours. During this period, approximately 76 workers were intermittently in the area where the exposed source was located.

A pipefitter noticed the source on the floor and picked it up; however, he dropped it when he suspected its nature and immediately called the radiographer. The radiographer picked up the source by the pigtail (flexible cable attached to the source) and manually inserted it into the radiography camera he was using.

Nature of Exposure or Loss

Most of the persons who were exposed to the source while it was lost were construction contractor employees, who were not wearing film badges or pocket dosimeters. Exposure estimates for all individuals involved ranged from a few 10's of millirem to approximately 11 to $15 \mathrm{rem}$ whole body. (Five individuals exceeded $3.0 \mathrm{rem}$ per calendar quarter).

\section{Thyroid Exposures}

Mercury, Nev., June 6, 1963 -Ref: 63-26

Nature of Incident

Group of workers exposed to airborne concentrations of radioiodines. 
Reentry and recovery operation being conducted in conjunction with detonation of nuclear device underground.

Details of Incident

The blast from the detonation caused a damper unit controlling the ventilation in the recovery drift to close partially, thereby reducing the amount of ventilation in the drift. The partial closing of the damper was not observed nor detected until tunnel operations had been suspended; therefore, the tunnel reentry party entered the tunnel. No respiratory equipment was used, since it had been previously determined that none was required. The presence of radioactive gas was not detected until the filter cartridge used in the air filter was measured.

Nature of Exposure or Loss

Fifteen workers were exposed to airborne concentrations of radioiodines resulting in estimated thyroid exposures ranging from 6 to $89 \mathrm{rem}$. Thirteen of these men received exposures in excess of the reportable 30 rem per year to thyroid.

\section{Leak of Radioactive Solution}

Golden, Colo., June 20, 1963 -Ref: $63-32$

Nature of Incident

Leak developed in overhead transfer line.

Description of Operation

Transferring radioactive solutions from one laboratory to another by stainless steel line.

Details of Incident

A leak in a stainless steel line carrying plutonium solution over the false ceiling of a laboratory room was discovered after the contaminated solution had spread throughout the perforated panel ceiling, over the walls and onto the floor. The leak was due to a chemical reaction between the liquid and the line.

Nature of Exposure or Loss

No exposures resulted from the incident. Decontamination cost was $\$ 8,364$.

\section{Hand Exposures}

Berkeley, Calif., June 28-July 8, 1963-Ref: $63-35$

Nature of Incident

Two employees received quarterly hand exposure exceeding 25 rems.

\section{Description of Operation}

Physical transfer of radioactive sources by use of tongs. 
Details of Incident

During the transfer of radioactive sources from a small carbon or tantalum oven into a high-vacuum chamber, two employees received hand exposures. The transfer was made in the box by the use of short tongs and not the standard manipulators installed in the box.

Nature of Exposure or Loss

The employees received hand exposures of $54.1 \mathrm{rem}$ and $44 \mathrm{rem}$. They did not exceed the annual hand exposure of $75 \mathrm{rem}$. Their total annual hand exposures were $72.9 \mathrm{rem}$ and 64.2 rem.

\section{Tritium Release}

Aiken, S. C., July 23, 1963 - Ref: 63-43

Nature of Incident

Accidental release of tritium to atmosphere through stack.

Description of Operation

Plant's separation facilities.

Details of Incident

Tritium had collected in the exhaust pumping system and was purged to the stack by a subsequent flow of inert gas. The tritium entered the pumping system either through a leaking valve or by misoperation of a valve.

Nature of Exposure or Loss

There were no detectable assimilations by personnel and no area contaminations were noted.

\section{$X$-Ray Exposure}

Lynchburg, Va., July 25, 1963 -Ref: $63-44$

Nature of Incident

Employee accidentally exposed to X-ray radiation during routine production activities.

Description of Operation

Operation of $\mathrm{X}$-ray machine used for fuel plate radiography.

Details of Incident

An employee entered an X-ray room, not knowing that an automatic interlock system had malfunctioned. The interlock system, when functioning normally, prevented the X-ray room door from opening when the X-ray machine was in operation. Upon entering the room, the employee was exposed. 
The amount of exposure received by the employee, as indicated by his film badge, was 6 rem whole body.

\section{Minor Contamination Incident}

Pocatello, Idaho, Aug. 3, 1963 -Ref: 63-39

Nature of Incident

Leakage of contaminated liquid from container dropped at terminal.

Description of Operation

Transfer of container during shipment.

Details of Incident

A 3,250-1b. cask, containing six fuel specimens, was dropped while being transferred at an intermediate terminal. Approximately two ounces of liquid leaked from the container, contaminating a $1 / 2$-sq. -ft. spot on a wooden dock $(1 / 2$ to $1 \mathrm{mr} / \mathrm{hr}$ at contact).

Nature of Exposure or Loss

Decontamination was effected by removing $1 / 8$ th in. of the wooden surface. There were no exposures nor was the cask damaged.

\section{Synchrotron Exposures}

Argonne, Ill., Oct. 17, 1963-Ref: 63-60

Nature of Incident

Employee received whole-body exposure exceeding 3 rem per calendar quarter.

Description of Operation

Making adjustment on gradient synchrotron.

Details of Incident

Before the work of adjusting the synchrotron began, a beam stopper was inserted into the synchrotron to prevent exposure to the employees; however, the correct beam stopper was not inserted. Unaware of this, the employees carried out their assignment and, therefore, were exposed.

Nature of Exposure or Loss

Two employees received whole-body exposures of $3.8 \mathrm{rem}$ and $2.8 \mathrm{rem}$ as a result of this incident.

\section{Tritium Exposures}

Oak Ridge, Tenn., Oct. 28, 1963-Ref: $63-46$ 
Nature of Incident

Exposure to tritium oxide resulting in a body burden of $90 \mathrm{mc}$.

Description of Operation

Modification of chemical hood.

Details of Incident

An employee was working in a chemical hood, replacing two silver chloride windows of an infrared absorption cell from which tritium oxide had been removed six days previously. A fraction of the residual tritium oxide which had become "fixed" to the interior surfaces of the cell diffused out of the hood and into a laboratory during performance of the work.

Nature of Exposure or Loss

The exposure was from a single intake of tritium which resulted in an estimated total radiation dose (for tritium) to the total-body tissue of $12.5 \mathrm{rem}$.

General contamination of the laboratory and adjoining corridor occurred; however, these areas were readily decontaminated.

\section{Beta Exposures}

Los Alamos, N. Mex., Oct.-Dec. 1963-Ref: 63-62

Nature of Incident

Two employees received whole-body skin doses in excess of $10 \mathrm{rem}$ per calendar quarter.

Description of Operation

Casting.

Details of Incident

During the casting operation of normal uranium, two men received whole-body beta skin doses.

Nature of Exposure or Loss

The employees received beta exposures of 15.17 and $17.29 \mathrm{rems}$ to the skin of the whole body.

\section{Fire Spread Contamination}

Richland, Wash., Nov. 6, 1963-Ref: 63-48

Nature of Incident

Fire and reaction of nitric acid with plutonium ion exchange resin.

Description of Operation

Plutonium processing facility. 
A sudden reversal of airflow in a plutonium purification facility resulted from the venting of an overpressurized anion-exchange vessel through a flange with a ruptured gasket. There was a swishing sound, some vibration, and instrument case covers on the panel board popped open, emitting dust into the room. Three operators on duty evacuated immediately. There was no fire detection system in the viewing room in which the fire started. The facility was shut down, after which two radiation monitors cautiously entered the area. They noted the reflection of flames shining through a partially opened door. Because of criticality considerations, only dry chemical extinguishers were used to combat the fire, which was brought under control in about $1 \frac{1}{2}$ hours.

As a consequence of the fire, alpha contamination was spread rather widely throughout the building. Areas surrounding the building were immediately placed under stringent contamination control.

Nature of Exposure or Loss

After the fire, low-level external contamination detected on firemen was removed without difficulty. Internal and external dosimetry data confirmed that there were no significant exposures. The cost of the incident was $\$ 397,000$, the majority of which was due to cleanup, decontamination of facilities, and disposal of contaminated trash.

\section{Contamination Spread By Employees}

Idaho Falls, Idaho, Nov. 15, 1963 - Ref: $63-52$

Nature of Incident

Spread of plutonium contamination by employees.

Description of Operation

Routine welding operation in portable glovebox.

\section{Details of Incident}

Unaware that a portable glovebox was highly contaminated, two men (a fitter and a welder) proceeded to use the box for the welding of nonradioactive toxic material. (The glovebox had been used previously to weld capsules containing radioactive material and had not been decontaminated nor properly tagged with a label indicating the presence of contamination.) After finishing the welding in the glovebox, the two men moved to a new location, unaware of the presence of alpha contamination on them and their tools as a result of the welding operation in the glovebox. The first indication came two days later when the fitter brought his toolbox from another job, where there was a known possibility of contamination, to the health physics office to have his tools checked for contamination, as required.

\section{Nature of Exposure or Loss}

All of the contamination was in very low-level amounts and internal and external dosimetry data confirmed that there were no significant exposures to personnel. Minor amounts of easily removable contamination were found throughout the plant area and offsite. Actual costs associated with the incident were approximately $\$ 25,000$.

\section{Release of Radioactive Material}

Miamisburg, Ohio, Dec. 21, 1963-Ref: 63-61 
Nature of Incident

Accidental release of radioactive material in building.

Description of Operation

Laying copper tubing service line in crawl space for ultimate use in two laboratory rooms.

Details of Incident

While installing a copper tubing service line in a crawl space, two maintenance men, with their view blocked, ruptured a vinyl bag with the tubing, releasing radioactive material.

The vinyl bag was wrapped around the end of a portion of an obsolete stainless steel pipe, formerly used as a vacuum transfer system for transferring radioactive solutions from one laboratory to another.

Nature of Exposure or Loss

Internal dosimetry data confirmed that there were no significant systemic body burdens resulting from this incident. The total cleanup cost was $\$ 7,611$.

No radioactive material was released outside the building.

\section{Minor Contamination Incident*}

Richland, Wash., Jan. 23, 1964-Ref: 64-2

Nature of Incident

Several employees received minor contamination while replacing equipment in waste storage facilities.

Description of Operation

Modification of waste storage facilities.

Details of Incident

While employees were replacing an agitator on a waste storage tank containing crude strontium, convection currents carried the heated contaminated vapor from the open top of the tank to the immediate environs.

Nature of Exposure or Loss

Internal and external dosimetry data confirmed that there were no significant radiation exposures. Minor skin and clothing contamination was quickly washed away.

The cost of the incident was $\$ 2,141$.

\section{Whole-Body Exposure}

Columbus, Ohio, Jan.-Mar. 1964-Ref: 64-14

Nature of Incident

Employee received whole-body exposure exceeding reportable 3 rem per calendar quarter.

*Included only because a press release was issued. 
Description of Operation

Hot cell facility.

Details of Incident

The exposure occurred when a technician was doing routine work at a hot cell facility. The cause of the exposure, from mixed fission products, was failure to keep proper account of the employee's radiation exposure history.

Nature of Exposure or Loss

A technician received a quarterly external whole-body gamma exposure of $3.25 \mathrm{rem}$.

\section{Exposure Incident}

Shippingport, Pa., Jan.-Mar. 1964-Ref: 64-21

Nature of Incident

Two employees received whole-body exposures exceeding reportable $3 \mathrm{rem}$ per calendar quarter.

Description of Operation

Reactor refueling operation.

Details of Incident

The exposures were due to an error in adding film badge data.

Nature of Exposure or Loss

Two employees received whole-body exposures of $3.26 \mathrm{rem}$ and $3.12 \mathrm{rem}$, respectively.

\section{Line-Block In Reactor}

Aiken, S. C., Feb. 15, 1964-Ref: $64-20$

Nature of Incident

Temporary line-block left in process piping following repair and maintenance work on reactor steam generator.

Description of Operation

Test reactor shutdown for maintenance work.

Details of Incident

During nonnuclear test operation of a test reactor at a moderator temperature of $165^{\circ}$ on February 24, erratic flows that had been observed just previously culminated in a rapid decrease in flow rate from $10,000 \mathrm{gpm}$ of $\mathrm{D}_{2} \mathrm{O}$ through the reactor to about $8,700 \mathrm{gpm}$. Subsequent investigation determined that a temporary line-block had been left unintentionally in the process piping during maintenance work on February 14. The plywood line-block was disintegrated by the combined flow and temperature of the system. Pieces of these parts caused partial blockage of the muff screens which protect each of 34 test and driver fuel positions in the re- 
actor, and soluble components caused some discoloration and turbidity of the $\mathrm{D}_{2} \mathrm{O}$ moderatorcoolant. Most of the material in the original line-block was collected on these screens, and was removed and accounted for by removing and cleaning the screens after each of several periods of maximum flow through the reactor. The soluble and suspended matter in the moderator was removed by filtration and ion exchange. These measures returned the reactor to its previous condition by March 16.

Nature of Exposure or Loss

The loss charged to this incident, $\$ 10,460$, represents the cost of materials and labor used to restore the moderator to pre-accident purity.

\section{Whole-Body Exposure}

Berkeley, Calif., Feb.-Mar. 1964-Ref: 64-60

Nature of Incident

Employee's film badge indicated whole-body exposure exceeding reportable 3 rem per calendar quarter.

Description of Operation

Routine laboratory work.

Details of Incident

Investigation revealed that the employee was not involved in an incident; however, in the absence of positive information supporting nonexposure, it was assumed that the person involved was exposed.

Nature of Exposure or Loss

Employee's film badge indicated an exposure of $8.2 \mathrm{rem}$ whole body.

\section{Hand Exposure}

Richland, Wash., Mar. 11, 1964-Ref: 64-11

Nature of Incident

Employee received hand exposure exceeding reportable 25 rem per calendar quarter.

Description of Operation

Removing material from storage basin of reactor.

\section{Details of Incident}

While an employee was removing material from a reactor storage basin, a short piece of radioactive wire pierced his glove (unknown to him) and lay against his thumb for a short time. The wire, used to wrap fuel element bundles, did not penetrate his skin.

Nature of Exposure or Loss

The employee received a hand exposure of $57 \mathrm{rem}$. 


\section{Contamination Incident}

Santa Susana, Calif., Mar. 19, 1964-Ref: 64-13

Nature of Incident

Contamination of asphalt surface.

Description of Operation

Radioactive liquid waste handling system.

Details of Incident

Clean water, being used to test two new waste tanks, was contaminated by condensation from a contaminated vent line connected to one of the tanks. The water was subsequently drained onto an asphalt surface, contaminating it and a drainage ditch.

Nature of Exposure or Loss

Some detectable contamination was found on a few employees; however, there was no personnel exposure problem. The contaminated personnel, the asphalt area and the equipment were readily decontaminated.

The cost of cleanup was approximately $\$ 6,000$.

\section{Reactor Charging Error}

Richland, Wash., Apr. 17, 1964-Ref: 64-39

Nature of Incident

Charging error made in loading reactor.

Description of Operation

Reactor was being restarted after a shutdown during which a fuel discharge-recharge operation and process tube replacement occurred.

\section{Details of Incident}

Shortly after startup, reactivity anomalies were observed. Efforts were made to continue operation, controlling the reactor with rods and shims, but when the reactivity was properly adjusted, calculations showed the flux was improperly peaked. The reactor was shut down and a second charge-discharge operation was carried out. It was found that an error had been made in the pattern of fuel, target, and mixer elements loaded into the reactor.

Nature of Exposure or Loss

Cost of $\$ 51,300$ was due to the reprocessing of the mischarged fuel elements.

\section{Truck Incident}

Kansas City, Mo., Apr. 22, 1964-Ref: 64-18

Nature of Incident

55 -gallon drum, containing 5 polyethylene bottles of radioactive material, damaged during transit. 
Description of Operation

Truck shipment of radioactive materials.*

Details of Incident

During transit, the ring bolts became loosened on two drums (lying on their sides) and the lids came off, allowing 4 bottles containing $93 \%$ enriched uranium to escape from one drum.

Nature of Exposure or Loss

There were no personnel exposures, nor was there any spillage of material or contamination as a result of the incident.

\section{Tritium Release}

Aiken, S. C., Apr. 23, 1964-Ref: 64-22

Nature of Incident

Accidental release of tritium to atmosphere through stack.

Description of Operation

Plant's separation facilities.

Details of Incident

Tritium had apparently been oxidized to water over a long period of time and the water had collected in a pipeline. The presence of the tritiated water in the pipeline was completely unexpected. During a routine maintenance operation, the tritiated water sprayed out into the process hood and could not be recovered.

Nature of Exposure or Loss

The maintenance mechanic who opened the line was wearing a plastic suit, as specified for this type of work, which probably prevented a significant tritium assimilation.

\section{Polonium Exposure}

Miamisburg, Ohio, Apr. 27-May 11, 1964-Ref: 64-36

Nature of Incident

Chemist received body deposition of polonium 210 .

Description of Operation

Polonium processing line.

*During 1964, there were six incidents involving trucks or trains transporting radioactive materials, in which no radioactive material was released and there was no loss of integrity to the package. In three additional incidents, radioactive material was released, but confined to the package or vehicle. (See TID-16764, Suppl. 2, for details). 


\section{Details of Incident}

Two separate events occurred in a period of ten days which could have caused the exposure:

1. Spill of contaminated water onto a laboratory floor (the chemist was present at the time of the incident).

2. Entry by the chemist, in protective clothing, to the high-risk area behind the polonium process lines to clean out several hoods.

It was not possible to determine which event produced the exposure; however, both may have contributed to the increased body burden of polonium.

\section{Nature of Exposure or Loss}

This internal deposition of polonium $(0.4 \mu \mathrm{c}$ total body) was detected through the routine urine specimens for polonium analysis. Organ doses for ensuing year from average burden are: spleen, $60 \mathrm{rem}$; kidney, $45 \mathrm{rem}$. Reportable yearly doses are $15 \mathrm{rem}$ per organ.

\section{Cyclotron Exposure}

Berkeley, Calif., Apr.-June 1964-Ref: 64-46

Nature of Incident

Employee received whole-body exposure exceeding 3 rem.

Description of Operation

Cyclotron bombardment of materials.

Details of Incident

While removing targets, which were bombarded by a cyclotron, from a vacuum chamber in a high-level cave, an employee was exposed to radiation.

Nature of Exposure or Loss

Employee received a whole-body dose of $3.4 \mathrm{rem}$.

\section{Minor Release of Fission Products}

Idaho Falls, Idaho, May 10, 1964-Ref: 64-19

Nature of Incident

Release of radioactive fission products at waste plant tank farm.

\section{Description of Operation}

Waste plant tank farm.

\section{Details of Incident}

The incident occurred when a steam-flushing operation was underway to remove radioactive contamination from three pipelines to permit their tie-in to new lines. During this operation, a leak developed in a hose coupling. Contaminated fluid issued from this leak, along with steam, and was rapidly dispersed by a high wind over an area of approximately three 
acres inside the plant fence. Contamination above background was found over an area of approximately ten acres outside the plant fence.

Investigation of the incident revealed that the inlet valves on the lines intended to be steamed were mislabeled so that steam was being put into a line which was not open.

Nature of Exposure or Loss

Internal and external dosimetry data confirmed that there were no significant radiation exposures. The cost of the incident was $\$ 12,884$, the majority of which was due to cleanup.

\section{Minor Contamination Incident}

Pocatello, Idaho, May 18, 1964-Ref: 64-23

Nature of Incident

Leakage of contaminated water from dropped container.

Description of Operation

Truck shipment of radioactive material.

Details of Incident

At arrival, a routine monitoring check of a cask revealed that the cask was reading $6 \mathrm{r} / \mathrm{hr}$ (gamma) from the top. Investigation revealed that the cask had been dropped on its side at an intermediate terminal prior to loading on the truck trailer.

Nature of Exposure or Loss

There were no significant personnel radiation exposures as a result of this incident nor was ther $€$ any contamination of the involved truck and trailer. A survey of the dock indicated a 10-square-foot contaminated area with readings to $20 \mathrm{mr} / \mathrm{hr}$ and several isolated spots reading $2 \mathrm{mr} / \mathrm{hr}$. These were readily decontaminated to levels below $1 \mathrm{mr} / \mathrm{hr}$.

\section{Tritium Release}

Aiken, S. C., June 3, 1964-Ref: 64-28

Nature of Incident

Accidental release of tritium to atmosphere through stack.

Description of Operation

Plant's separation facilities.

Details of Incident

While a reservoir was being pinch-welded, the welding machine malfunctioned, causing the side of the reservoir seal tube to blow out, thereby releasing the gas.

Nature of Exposure or Loss

There were no detectable assimilations by personnel and no area contaminations were noted. 


\section{Glovebox Explosion}

Golden, Colo., June 12, 1964 -Ref: 64-25

Nature of Incident

Explosion in glovebox during plutonium chip-degreasing operation.

Description of Operation

Plutonium process line.

Details of Incident

An explosion occurred during a plutonium chip-degreasing operation which involved the dipping of plutonium chips, contained in a stainless steel can with a perforated bottom, into a carbon tetrachloride bath. The operator dipped the chips, hung them on a hook to dry, then left the glovebox to get more chips. When he returned, less than five minutes later, the chips were on fire. The procedure for handling burning plutonium under these conditions is to put the chips on the floor of the glovebox and to cover them with another can until they burn out. The operator reached into the glovebox with his left hand in a rubber glove to do this. The can of burning chips slipped from his hand and fell into the carbon tetrachloride bath. An explosion resulted immediately.

Nature of Exposure or Loss

The left thumb and index finger of the operator were removed to effect decontamination because small particles of plutonium had become embedded in them.

The total direct cost due to equipment and material loss and to decontamination was $\$ 56,400$.

\section{Hand Exposures}

Notre Dame, Ind., June 18, 1964 -Ref: $64-30$

Nature of Incident

Two employees received finger exposures.

Description of Operation

Repairing 2000-curie cobalt 60 unit.

\section{Details of Incident}

While a cobalt 60 unit was being repaired, the source, which is attached to a $3 / 8$-inch diameter vertical rod, became detached from the rod. In an attempt to retrieve the source, which had fallen into a shielded cavity, by "fishing" through the $1 / 2$-inch diameter hole in the turret with a $1 / 8$-inch diameter retrieval rod, two employees were exposed to radiation.

Measurements of the dose rate from the hole were taken before the fishing operation began; however, they were made incorrectly. Unaware of the true measurements, the operation proceeded.

Nature of Exposure or Loss

Assuming a maximum exposure time of one hour, both employees received an integrated dose of $180 \mathrm{rem}$ to the tips of their fingers. 


\section{Tritium Release}

Aiken, S. C., June 19, 1964 -Ref: $64-34$

Nature of Incident

Accidental release of tritium to atmosphere through stack.

Description of Operation

Plant's separation facilities.

Details of Incident

The tritium was released unknowingly from a hold tank to the stack piping as a result of valve malfunction or operator error.

Nature of Exposure or Loss

There were no detectable assimilations by personnel and no area contaminations were noted.

\section{Tritium Release}

Aiken S. C., June 28, 1964-Ref: 64-33

Nature of Incident

Accidental release of tritium to atmosphere through stack.

Description of Operation

Plant's separation facilities.

Details of Incident

The release occurred when a protective rupture disc on a low-pressure process pipeline cracked slightly and allowed the tritium to flow through into the relief system.

Nature of Exposure or Loss

There were no detectable assimilations by personnel and no area contaminations were noted.

\section{Contamination Incident}

Apollo, Pa., July 10, 1964-Ref: 64-35

Nature of Incident

Trailer transporting radioactive material contaminated.

Description of Operation

Truck shipment of thorium nitrate tetrahydrate in fiberboard drums (60). 


\section{Details of Incident}

Upon arriving at destination, one drum was found to be contaminated and to have lost a small amount of its contents. The drums had been moved via two trailers.

Nature of Exposure or Loss

There were no personnel exposures. The trailers were found to have minor contamination which was readily removed.

\section{Plutonium Deposition Incident}

Richland, Wash., July 10, 1964-Ref: $64-37$

Nature of Incident

Fragment of plutonium metal struck technician in upper left arm.

\section{Description of Operation}

Routine metallurgical tests on plutonium metal specimens of various thicknesses being performed in glovebox.

\section{Details of Incident}

A plutonium specimen was placed between the anvils of a hydraulic press (housed in the inert atmosphere of a glovebox) to reduce the specimen's thickness. During the operation, pressure was applied over a period of time and, without warning, the plutonium specimen disintegrated with a rifle-like sound, ejecting incandescent fragments, some being thrown outside the inert gas atmosphere within the glovebox.

\section{Nature of Exposure or Loss}

One employee received an internal deposition of plutonium in the range of one to ten body burdens (maximum permissible body burden for plutonium 238, with bone as reference, is 0.04 $\mu c)$, as a result of a plutonium fragment lodging in his arm.

Minor contamination of the adjacent working area occurred resulting in cleanup costs of $\$ 750$.

\section{Hand Exposure}

Idaho Falls, Idaho, July 22, 1964-Ref: $64-40$

\section{Nature of Incident}

Employee received hand exposure of $31 \mathrm{rem}$ to one hand and $5 \mathrm{rem}$ to the other.

\section{Description of Operation}

Experiment involving removal of radioactive wires from stainless steel tubes.

\section{Details of Incident}

This exposure was received while performing work involving the removal of flux wires from stainless steel tubes, a routine operation associated with an experiment. On this particular occasion, however, the flux wire was bent and required straightening, which was done 
manually with the wire submerged under water. The manual handling of the wire was the result of an error in judgment.

Nature of Exposure or Loss

The employee received approximately $31 \mathrm{rem}$ of penetrating radiation to one of his hands and 5 rem to the other.

\section{Rail Incident}

Madison, Ill., July 23, 1964-Ref: $64-38$

Nature of Incident

Two drums, containing 0.947 enriched uranium, fell out of railroad car.

Description of Operation

Transporting 251 drums, containing 0.947 enriched uranium residues, by rail.

Details of Incident

During railroad yard switching operation, a railroad car door broke open and two drums fell out. Part of the contents of one of the drums spilled.

Nature of Exposure or Loss

There were no personnel exposures. A radiological assistance team (AEC) cleaned up the area, replacing the material and sealing the drum. The railroad car was decontaminated.

\section{Tritium Release}

Aiken, S. C., July 24, 1964-Ref: $64-53$

Nature of Incident

Accidental release of tritium to atmosphere through stack.

Description of Operation

Plant's separation facilities.

Details of Incident

Tritium was pumped inadvertently from the process system into a pipeline connected to the stack.

Nature of Exposure or Loss

There were no detectable assimilations by personnel and no area contaminations were noted.

\section{Contaminated Cask}

Middletown, Conn., Sept. 2, 1964-Ref: 64-47 
Nature of Incident

Exterior of shipping cask contaminated.

Description of Operation

Truck shipment of cask carrying irradiated shim motors.

Details of Incident

During routine monitoring at destination, contamination (fission products) was detected on the outside of a cask and its supports and also on the floor of the trailer. The cask was in the front part of the trailer during the entire shipment; therefore, there was no contamination of other cargo being transferred and/or delivered.

Nature of Exposure or Loss

There were no significant personnel radiation exposures as a result of this incident. The trailer bed was contaminated to a level of 5 to $10 \mathrm{mr} / \mathrm{hr}$ over an area of several square feet where the cask was located during shipment. The trailer was readily decontaminated.

\section{Hot Canyon Fire}

Aiken, S. C., Oct. 1, 1964-Ref: 64-54

Nature of Incident

Anion resin fire in hot canyon.

Description of Operation

Remote repair work to remove residue plugging an anion exchange column piping.

Details of Incident

During a remote repair operation, a crane operator observed a white vapor, which he believed to be steam (later determined to be smoke from a fire), emerging from around the base of an anion exchange column used for the recovery of neptunium from high-activity waste. This fire gave rise to airborne contamination that grossly contaminated the outside of a hot canyon crane used for remote maintenance and penetrated the crane cab air-conditioner filters, causing low-level contamination of the crane cab.

Nature of Exposure or Loss

The crane operator had minor skin and nasal contamination.

The cost of the incident, including decontamination of the crane, was $\$ 21,000$.

\section{Glovebox Explosion}

Miamisburg, Ohio, Oct. 27, 1964-Ref: 64-55

Nature of Incident

Explosion in glovebox line.

Description of Operation

Glovebox process line. 
Details of Incident

Ignition by unknown sources of a methanol-air mixture led to an explosion within a glovebox line in which 17 grams of plutonium 238 were being processed. No one was injured; however, the explosion pressurized the boxes and caused a total of six gloves to be torn from their ports.

Nature of Exposure or Loss

The clothing and external body surfaces of three employees were contaminated. All three men received less than a body burden $(0.04 \mu \mathrm{c})$ of plutonium.

Plutonium oxide discharged from the open gloveports and spread throughout the operating areas of the building. Decontamination costs, $\$ 33,722$; property damage, $\$ 1,200$.

\section{Container Leakage}

Chicago, Ill., Nov. 11, 1964-Ref: $64-57$

Nature of Incident

Contaminated liquid leaked from shipping container.

\section{Description of Operation}

Truck shipment of cask carrying nine unclad fuel pins ( 490 grams of $20 \%$ enriched uranium).

\section{Details of Incident}

Upon arrival at destination, the shipment (an autoclave housed in a lined shipping cask and containing nine unclad fuel pins in $432 \mathrm{cc}$ 's of water) was discovered to have leaked during transit. The leak occurred when a broken valve allowed contaminated water inside the autoclave to leak into the shielded container and then seep out onto the floor of the truck. The cask was transported on three different trailers between origin and destination.

\section{Nature of Exposure or Loss}

Only one of the trailers that hauled the cask was found to be contaminated $\left(3^{\prime} \times 6^{\prime}\right.$ area). This area of the floor was replaced.

Minor contamination was found on the outside of several cartons of the cargo hauled in the contaminated trailer.

There were no radiation exposures nor any personnel contamination.

\section{Tritium Release}

Aiken, S. C., Dec. 22, 1964-Ref: 64-66

Nature of Incident

Accidental release of tritium to atmosphere through stack.

Description of Operation

Plant's separation facilities.

Details of Incident

The tritium loss was caused by an unexpected cladding failure on a fuel element. 
Nature of Exposure or Loss

There were no personnel exposures nor any areas contaminated.

\section{Lung Exposure}

Oak Ridge, Tenn., Dec. 1964-Ref: 64-69

Nature of Incident

Employee received lung exposure.

Description of Operation

Uranium recovery.

Details of Incident

While sampling, weighing, and grinding uranium oxides, of varying assays, in an enclosed process system, an operator received a lung burden of $0.046 \mu \mathrm{c}$.

Nature of Exposure or Los's

The employee had integrated lung dose of 19.4 rem for the first year.

No other employees exceeded one-half of the permissible body burden. 


\section{ACCIDENTS INVOLVING FATALITIES IN AEC ACTIVITIES 1963-1964}

\section{Fall Into Maintenance Cell}

Lincoln, Nebr., Jan. 5, 1963 - Ref: 63-2

Nature of Accident

Fall into open shield plug hole.

Description of Operation

Removing source cask from floor of maintenance cell to floor of reactor room by high bay crane.

\section{Details of Accident}

While giving signals to the operator of the high bay crane, the shift supervisor, while walking backward, fell 54 feet through an open shield plug hole to the floor below.

Nature of Injuries or Loss fall.

The shift supervisor died of internal injuries and a basal skull fracture resulting from the

\section{Caught Between Vehicle And Dock*}

Portsmouth, Ohio, Jan. 14, 1963

Nature of Accident

Pinned between rear of truck and loading dock.

Description of Operation

Delivering tires.

\section{Details of Accident}

A vendor's truck driver had unloaded tires from his truck to the loading dock. After closing the tailgate on the truck, the truck rolled backward, pinning the driver against the dock.

* This fatality is included in this publication because it was associated with an AEC activity and a member of the public. However, for statistical purposes, it is not included in our overall figures. 
The truck driver died of internal injuries later in the day.

\section{Crushed By Mobile Equipment}

Hattiesburg, Miss., Feb. 16, 1963 -Ref: 63-11

Nature of Accident

Caterpillar tractor ran over employee.

Description of Operation

Clearing of area for placement of equipment.

Details of Accident

A construction worker, for some unknown reason, walked into the path of a tractor, as its operator was backing up to push dirt. The operator was concentrating on missing a piece of pipe lying in the path of the tractor and did not see the construction worker.

Nature of Injuries or Loss

The construction worker was crushed to death when the entire length of the tractor tread ran over his body, causing multiple internal injuries.

\section{Electrocution}

Oak Ridge, Tenn., Apr. 30, 1963 -Ref: 63-24

Nature of Accident

Electrocution.

Description of Operation

Testing of a diagnostic ion source being developed in laboratory for experimental use.

\section{Details of Accident}

A senior physicist was conducting an experiment utilizing a complex array of equipment, with the power supply connected to the ion source supply cabinet. The physicist reached into the open portion of the cabinet and fell to the floor.

Nature of Injuries or Loss

The physicist received a fatal electric shock.

\section{Fall From Cooling Tower}

Idaho Falls, Idaho, June 25, 1963-Ref: 63-29

Nature of Accident

Fall. 
Description of Operation

Construction of fan cylinder on cooling tower.

Details of Accident

A carpenter, while attempting to align vertical staves during the construction of a fan cylinder on a cooling tower, lost his foothold and fell backward 65 feet to the cooling tower basin bottom.

Nature of Injuries or Loss

The carpenter was fatally injured. The cause of death was a compound fractured skull, broken neck and multiple internal injuries with massive hemorrhage.

\section{Fatal Fall}

Middletown, Conn., June 25, 1963 -Ref: $63-30$

Nature of Accident

Fall.

Description of Operation

Wooden boxes of waste being moved by forklift truck.

Details of Accident

An operator's helper was kneeling on the top of a box, being moved by a forklift truck, to counterbalance the overhanging weight, when the box tipped forward and he fell to the ground.

Nature of Injuries or Loss

The operator's helper died of a fatal pulmonary embolus 17 days later.

\section{Motor Vehicle Accident}

Mercury, Nev., July 10, 1963-Ref: $63-45$

Nature of Accident

Single car accident.

Description of Operation

Returning in a pickup truck from a job assignment.

Details of Accident

A custodial employee lost control of the pickup truck he was driving while attempting to light a cigarette. The vehicle rolled over and was severely damaged.

Nature of Injuries or Loss

The employee suffered two broken ribs and other internal injuries, was given medical treatment, and returned to work in a few weeks. Three months later, he died from a fatal pulmonary embolism believed to be the result of injuries (femoral vein thrombosis) received in the accident. 


\section{Asphyxiation}

Oak Ridge, Tenn., July 23, 1963-Ref: $63-36$

Nature of Accident

Asphyxiation.

Description of Operation

Testing piping system in valve pit.

Details of Accident

An apprentice pipefitter was checking for inert gas leaks in the piping system in a valve pit when he was overcome in an oxygen-deficient atmosphere.

Nature of Injuries or Loss

The apprentice pipefitter was asphyxiated. A pipefitter, attempting to save the life of the apprentice, was overcome by the gas but was subsequently revived.

\section{Fall From Scaffold}

Paducah, Ky., Aug. 1, 1963 -Ref: 63-41

Nature of Accident

Fall.

Description of Operation

Installation of mechanical equipment in water softening facility.

Details of Accident

While an ironworker was climbing down a rolling-type scaffold, the scaffold began to swing and fold, finally toppling to the floor. He held on until the scaffold was about halfway down, then fell the remaining distance to the floor.

Nature of Injuries or Loss

The ironworker died five days later as a result of multiple skull fractures.

\section{Chemical Burns}

Los Alamos, N. Mex., Sept. 26, 1963-Ref: 63-53

Nature of Accident

Second and third degree burns to $50-60 \%$ of body.

Description of Operation

Refuse dumping. 
When a truck backed up to deposit refuse in a dump, it ran over a 5 -gallon can containing a flammable liquid, believed to be paint thinner. The container ruptured and the contents sprayed out, saturating the clothing of a laborer standing eight to ten feet away. Smoldering embers in an adjacent debris fire caught his clothes on fire, engulfing him in flames.

Nature of Injuries or Loss

The laborer died about six weeks later as a result of second and third degree burns to $50-60 \%$ of his body.

\section{Motor Vehicle Accident}

Mercury, Nev., Mar. 11, 1964-Ref: $64-9$

Nature of Accident

Single car accident.

Description of Operation

Driving on official duty from one area to another.

Details of Accident

While driving alone on official duty, an electronics coordinator failed to negotiate a curve and the car overturned.

Nature of Injuries or Loss

The driver died as the result of a skull fracture received in the accident.

\section{Struck By Vehicle*}

Rincon, Puerto Rico, Mar. 12, 1964

Nature of Accident

Government-owned vehicle struck a member of the public.

Description of Operation

Transporting personnel.

Details of Accident

While a Government-owned motor vehicle was being driven through the city, a six-yearold boy darted from between two parked cars into its path.

Nature of Injuries or Loss

The boy died of internal injuries as a result of the accident.

* This fatality is included in this publication because it was associated with an AEC activity and a member of the public. However, for statistical purposes, it is not included in our overall figures. 


\section{Crushed By Water Tanker}

Mercury, Nev., Apr. 21, 1964-Ref: 64-15

Nature of Accident

Driver lost control of water tanker.

Description of Operation

Driving 8,000-gal. water tanker from loading station to area where roadbed was being sprinkled,

Details of Accident

Driver, for an unknown reason, lost control of 8,000-gal. water tanker which slid into a 12-foot ditch.

Nature of Injuries or Loss

The driver of the tanker was found crushed to death, with the left rear section of the tanker resting on the upper half of his body.

\section{Fall From Flat Roof}

Albuquerque, N. Mex., July 1, 1964-Ref: 64-31

Nature of Accident

Fall.

Description of Operation

Reroofing building.

Details of Accident

While laying roofing near the edge of a flat roof, a roofer fell 22 feet to the ground.

Nature of Injuries or Loss

The roofer died three days after he fell as a result of head and internal injuries sustained in the fall.

\section{Struck By Cable Spool}

Mer cury, Nev., Sept. 19, 1964-Ref: $64-49$

Nature of Accident

Cable spools jerked from racks striking four men.

Description of Operation

Electrical cables being lowered into underground complex. 
Electrical cables were being lowered into an underground complex when a wire rope from a hoist broke causing the cable spools to be jerked from their racks. The cables fell into the hole, clogging the shaft and trapping four men below ground.

Nature of Injuries or Loss

Four men (above ground) were hit by the spools, one receiving extensive head and chest injuries from which he died, the others receiving multiple contusions and abrasions. Four men trapped underground were rescued unharmed on the fourth day after the accident. Pxoperty damage $\$ 74,000$.

\section{Electrocution}

Menlo Park, Calif., Sept. 23, 1964-Ref: $64-50$

Nature of Accident

Electrocution.

Description of Operation

Drilling shafts through earth fill embankment covering accelerator housing.

\section{Details of Accident}

The drill rig operator was moving the mast (boom) from a horizontal to a vertical position when it contacted a high-voltage line overhead causing the current to flow through the drill rig to the ground.

Nature of Injuries or Loss

A laborer, apparently in contact with the rig, was electrocuted.

\section{Train Collision}

Richland, Wash., Dec. 8, 1964-Ref: 64-64

Nature of Accident

Locomotive and track maintenance car collided.

Description of Operation

Routine duties of the intraplant railroad.

Details of Accident

A locomotive and a maintenance car collided head-on.

Nature of Injuries or Loss

One man on the maintenance car died two weeks later from a fractured skull and brain injury received at the time of the accident; the second employee on the maintenance car was injured. Repair of the maintenance car cost approximately $\$ 500$. 


\section{Crushed By Falling Pipe}

Mercury, Nev., Dec. 15, 1964-Ref: 64-63

Nature of Accident

Struck by drill pipe.

Description of Operation

Routine drilling of emplacement hole.

Details of Accident

Failure occurred at the shank of the hook assembly, dropping $65,000 \mathrm{lbs}$ of metal. When it struck the rotary table, the drill pipe broke loose and continued on down the hole. The portion of the hook assembly or its appendages struck one man on the head and hit another man a glancing blow on his right arm, side and leg.

Nature of Injuries or Loss

One man was killed instantly from a crushing blow on the head. The other man received contusions and abrasions. Property damage, $\$ 2,340$. 